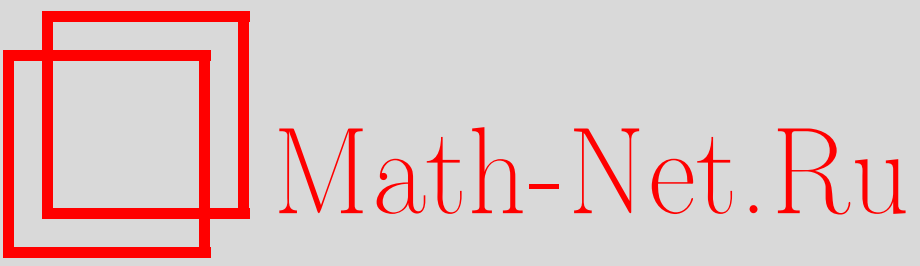

Я. Г. Синай, Аномальный перенос в почти-периодических средах, УМН, 1999, том 54, выпуск 1, 181-208

DOI: https://doi.org/10.4213/rm120

Использование Общероссийского математического портала Math-Net.Ru подразумевает, что вы прочитали и согласны с пользовательским соглашением

http://www.mathnet.ru/rus/agreement

Параметры загрузки :

IP : 54.147 .182 .235

26 апреля 2023 г., $11: 38: 35$ 


\title{
АНОМАЛЬНЫЙ ПЕРЕНОС В ПОЧТИ-ПЕРИОДИЧЕСКИХ СРЕДАХ
}

\author{
Я. Г. СинАй
}

СОДЕРЖАНИЕ

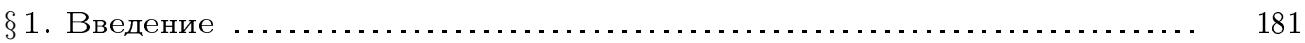

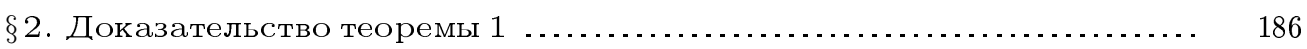

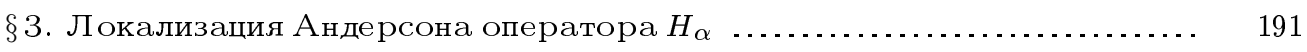

$\S 4$. Экспоненциальное убывание приближенных собственных функций и другие асимптотические соотношения ......................... 204

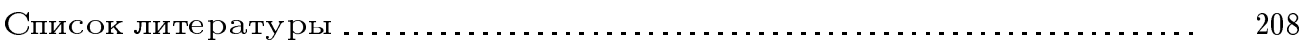

\section{$\S$ 1. Введение}

В этой работе мы обсуждаем новый тип случайных блужданий, отличающихся от броуновского движения или его модификаций. Впервые он был обнаружен в случайных блужданиях в случайных средах (см. [1]) и характерен тем, что траектория носит сильно выраженный флуктуационный характер: движущаяся точка застревает в "случайной яме" и колеблется около ее дна. Затем в достаточно большой момент времени, зависящий от глубины ямы, она перескакивает более или менее детерминированным образом в следующую яму, где ведет себя подобным образом, затем перескакивает в следующую яму и т. д. Такие блуждания естественно назвать флуктуационными. В [1] для флуктуационного блуждания требовалась определенная симметрия распределения вероятностей перехода. Ниже мы описываем другой класс задач, где флуктуационное блуждание появляется в открытой области значений параметров.

Обозначим через $\Omega_{t}$ ансамбль случайных траекторий $b_{t}=\{b(s), 0 \leqslant s \leqslant t\}$ длины $t$ таких , что $b(s+1)-b(s)= \pm 1,0$. Возьмем $C^{2}$-периодическую функцию $F$ периода 1 , имеющую две невырожденные критические точки, и положим $V(k, k+1)=$ $V(k+1, k)=0, V(k, k)=F(\omega k+\alpha)$, где $\omega-$ число врашения и $\alpha \in[0,1]-$ фаза. Введем статистический вес

$$
\pi\left(b_{t}\right)=\varepsilon^{\nu\left(b_{t}\right)} \exp \left\{\beta \sum_{s=0}^{t-1} V(b(s), b(s+1))\right\},
$$

где $\nu\left(b_{t}\right)=(k \mid 0 \leqslant k<t, b(k) \neq b(k+1)), \beta$ и $\varepsilon$ - параметры, и отвечающую ему 
статистическую сумму

$$
Z_{t}(\beta, \varepsilon)=\sum_{b_{t} \in \Omega_{t}} \pi\left(b_{t}\right)
$$

Траекторию случайного блуждания можно рассматривать как полимерную цеп, статистический вес (1) характеризует взаимодействие этой цепи со случайной средой. В нашем случае случайная среда является почти-периодической. Основные характеристики полимера описьваются средним квадратом смешения

$$
r_{t}=\frac{1}{Z_{t}(\beta, \varepsilon)} \sum_{b_{t} \in \Omega_{t}} b^{2}(t) \pi\left(b_{t}\right)
$$

и поведением при $t \rightarrow \infty$ распределения вероятностей

$$
\frac{1}{Z_{t}(\beta, \varepsilon)} \sum_{b_{t}: b(t)=x} \pi\left(b_{t}\right) .
$$

Ситуация с фиксированным $\varepsilon$ и малыми $\beta$ была исследована недавно Е. И. Динабургом (Динабург, частное сообщение), которьй показал, что при естественных предложениях $r_{t}$ растет при $t \rightarrow \infty$ пропорционально $t$, а указанное распределение вероятностей сходится к гауссовскому. Это означает, что при малых $\beta$ роль среды мало существенна, и статистика случайных траекторий такая же, как и в отсутствие внешнего поля.

Основное содержание этой работы посвящено противоположной ситуации, когда $\beta$ фиксировано, а $\varepsilon$ достаточно мало. Поэтому, не ограничивая обшности, можно считать $\beta=1$. Мы доказываем следующую теорему.

ТЕОрема 1. Для любого $\delta>0$ можнно выбрать

1) $\varepsilon_{0}(\delta)>0$

2) постоянные $0<c(\delta)<C(\delta)<\infty$;

3) челое число $t(\delta)$

так, что при любом $\varepsilon, 0<\varepsilon<\varepsilon_{0}(\delta), t>t(\delta)$ существуют подмножсество $R_{t} \in S^{1}, l\left(R_{t}\right) \geqslant 1-\delta, \quad$ и для всякого $\omega \in R_{t}$ подмножество $A_{t}(\omega) \in S^{1}$, $l\left(A_{t}(\omega)\right)>1-\delta$, такие, что для $\omega \in R_{t}, \alpha \in A_{t}(\omega)$

$$
c(\delta) t^{2 / 3} \leqslant r_{t} \leqslant C(\delta) t^{2 / 3}
$$

Для тех жее $(\omega, \alpha)$ найдется такая точка $x_{t}(\omega, \alpha)$, что для распределения вероятностей $P_{t}, P_{t}\left(b_{t}\right)=\frac{\pi\left(b_{t}\right)}{Z_{t}(\beta, \varepsilon)}$,

$$
P_{t}\left\{b_{t}:\left|b(t)-x_{t}(\omega, \alpha)\right| \geqslant u\right\} \leqslant C(\delta) e^{-\gamma_{0} u}
$$

где $\gamma_{0}=\gamma_{0}(\omega)>0$ ине зависит от $t u \alpha$.

Иными словами, распределение конца полимерной цепи $b(t)$, индуцированное $P_{t}$, экспоненциально локализовано в окрестности точки $x_{t}(\omega, \alpha)$, расстояние которой до нуля растет как $t^{1 / 3}$. Из этого утверждения, точнее, из его доказательства, можно 
вывести, что в условиях теоремы 1 полимерная цепь ведет себя, как траектория флуктуационного случайного блуждания. Пары $(\omega, \alpha)$, для которых выполнено утверждение теоремы 1 , естественно считать типичньми (для нашей задачи).

Доказательство теоремы 1 опирается на теорию локализации Андерсона (ЛА) для разностного оператора Шрёдингера с почти-периодическим потенциалом (см. [2]-[4]). Ниже в этом параграфе мы излагаем ряд первоначальных фактов, относяшихся к ЛА для указанного оператора. В $\S 2$ мы доказываем теорему 1, точнее, сводим ее к некоторьм оценкам. В $\S 3$ мы приводим подробньй обзор доказательства ЛА, а в $\S 4$ описываем, как получить нужные оценки. В целом $\S 3, \S 4$ покрывают нужды $\S 2$.

Одномерньй разностньй оператор Шрёдингера с почти-периодическим потенщиалом, который играет основную роль в этой работе, имеет вид

(2) $\quad\left(H_{\alpha} \Psi\right)(x)=\varepsilon \Psi(x-1)+\varepsilon \Psi(x+1)+\exp \{F(x \omega+\alpha)\} \Psi(x),-\infty<x<\infty$.

Легко видеть, что $H_{\alpha}$ - ограниченный самосопряженный линейный оператор в пространстве $l^{2}$ дважды бесконечных последовательностей $\Psi=\{\Psi(x),-\infty<x<\infty\}$. Справедливы следующие утверждения.

$1^{\circ}$. Для почти каждого $\omega$ и достаточно малых $\varepsilon,|\varepsilon| \leqslant \varepsilon_{0}(\omega)$, оператор $H_{\alpha}$ при почти всех $\alpha$ имеет чисто точечньй спектр. Именно это утверждение назьвается локализацией Андерсона.

$2^{\circ}$. Для каждой собственной функции (с.ф.) $\Psi_{\alpha, i}$ оператора $H_{\alpha}$ можно указать конечное множество $Z\left(\Psi_{\alpha, i}\right)$, назьваемое ее существенным носителем (с.н.), так, что $\Psi_{\alpha, i}$ сосредоточена в основном около с.н. $Z\left(\Psi_{\alpha, i}\right)$. Последовательное построение с.н. будет приведено в $\S 3$. Собственное значение (с.з.), отвечающее $\Psi_{\alpha, i}$, обозначается $\lambda_{\alpha, i}$.

$3^{\circ}$. Если $\Psi_{\alpha, i}$ есть с.ф. $H_{\alpha}$, то сдвинутая функция $\left(S \Psi_{\alpha, i}\right)(x)=\Psi_{\alpha, i}(x+1)$ есть c.ф. $\Psi_{\alpha+\omega, j}$ оператора $H_{\alpha+\omega}$. Сушественный носитель ковариантен по отношению к этой симметрии: $Z\left(S \Psi_{\alpha, i}\right)=Z\left(\Psi_{\alpha, i}\right)-1$.

Последнее свойство, разумеется, не определяет с.н. однозначно. Доказательство ЛА, которое мы имеем в виду (см. [4]), включает в себя построение с.н. для каждой с.ф. Из того, что $F$ принимает каждое значение не более чем в двух точках, можно вьвести (см. далее), что с.н. любой с.ф. содержит $2^{r}$ точек, $r \geqslant 0$, и имеет иерархическую структуру. А именно, если $\#\left(Z\left(\Psi_{\alpha, i}\right)\right)=2^{r}$, то $Z\left(\Psi_{\alpha, i}\right)$ состоит из двух непересекающихся подмножеств: $Z\left(\Psi_{\alpha, i}\right)=Z_{r, 1} \cup Z_{r, 2}, Z_{r, 2}=Z_{r, 1}+m_{r}$ для некоторого $m_{r}>0$. Множество $Z_{r, 1}$ таким же образом может быть разложено в сумму двух подмножеств $Z_{r, 1}=Z_{r-1,1} \cup Z_{r-1,2}, Z_{r-1,2}=Z_{r-1,1}+m_{r-1}$ и т. д. Типичньй вид с.н. представлен на рис. 1 для $r=0,1,2,3$. Схематичный вид с.ф. изображен на рис. 2. Сушественный носитель отмечен точками. Числа $m_{1}, m_{2}, \ldots, m_{r}$ связаны со структурой резонансов, которые отвечают каждой с.ф. $\Psi_{\alpha, i}$.

$4^{\circ}$. Обозначим через $\operatorname{pos}\left(\Psi_{\alpha, i}\right)$ крайнюю левую точку $Z\left(\Psi_{\alpha, i}\right)$ и введем множество $\Phi_{\alpha}$ тех с.ф. $\Psi_{\alpha, i}$, для которых $\operatorname{pos}\left(\Psi_{\alpha, i}\right)=0$. Пусть также $\Lambda_{\alpha}=\left\{\lambda \mid \Psi_{\alpha, i} \in \Phi_{\alpha}\right\}$. Функции $\Phi_{\alpha}$ и $\Lambda_{\alpha}$, вообше говоря, многозначные, а для некоторых $\alpha$ их значения могут быть пустыми множествами. В целом же как функции $\alpha$ они измеримы в естественном смысле этого слова, и для всего набора собственных функций $\left\{\Psi_{\alpha, i}\right\}$ имеет место соотношение

$$
\left\{\Psi_{\alpha, i}\right\}=\bigcup_{-\infty<m<\infty} S^{m} \Phi_{\alpha+m \omega} .
$$


$\circ$

- 0

- $\circ$

\section{Рис. 1}
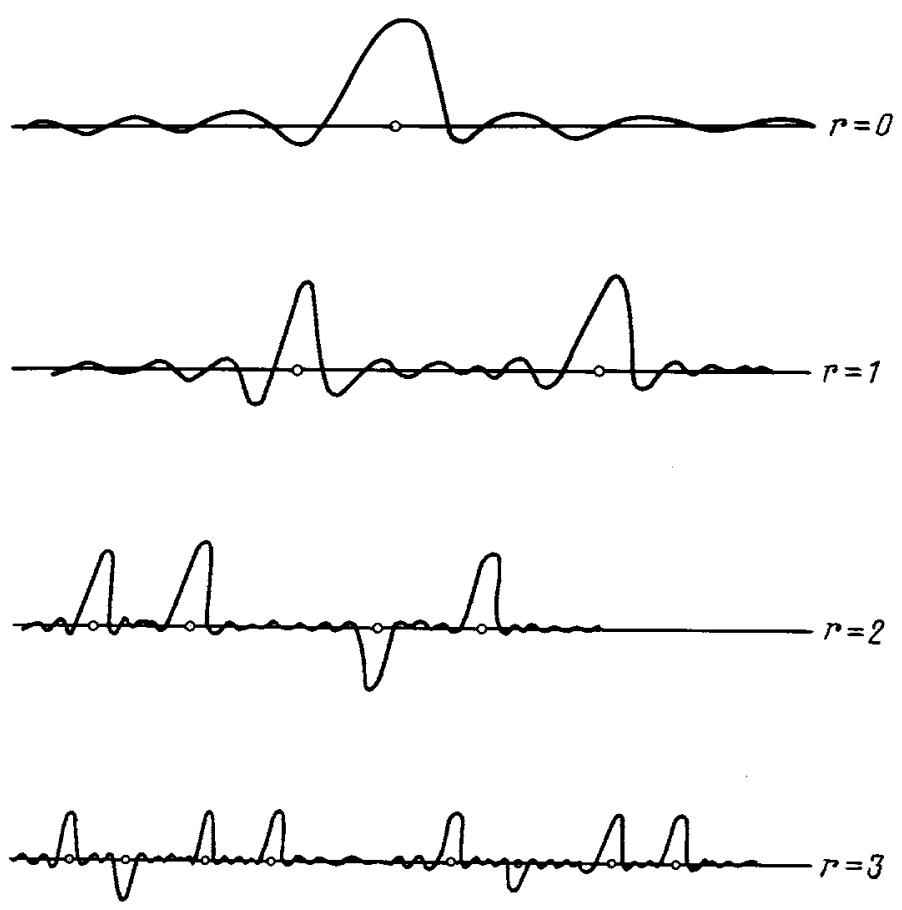

Рис. 2

Равенство (3) показывает, что весь базис с.ф. может быть выражен через значения измеримой функции $\Phi_{\alpha}$ вдоль траектории точки $\alpha \in S^{1}$ под действием поворота $T_{\omega}$ окружности $S^{1}$ на угол $\omega, T_{\omega} \alpha=\alpha+\omega(\bmod 1)$. Аналогичное соотношение справедливо для набора с.з.: если $\left\{\lambda_{\alpha, i}\right\}$ есть весь набор с.з. оператора $H_{\alpha}$, то

$$
\left\{\lambda_{\alpha, i}\right\}=\bigcup_{-\infty<m<\infty} \Lambda_{\alpha+m \omega}
$$

$5^{\circ}$. Пусть $M_{k} \subset S^{1}$ есть подмножество тех $\alpha$, где число с.ф. $\Psi_{\alpha, i}$, принадлежащих $\Phi_{\alpha}$, равно $k$. Тогда $l\left(M_{k}\right) \leqslant$ const $\rho^{k}$, где const и $\rho<1$ зависят от $\omega$ и $\varepsilon$ и стремятся к нулю при $\varepsilon \rightarrow 0$.

В действительности $l\left(M_{k}\right)$ убывают гораздо быстрее. 
$6^{\circ}$. Пусть $D_{k} \subset S^{1}$ есть подмножество тех $\alpha$, для которых $\Phi_{\alpha}$ содержит хотя бы одну с.ф. $\Psi_{\alpha, i}$, для которой $\operatorname{diam} Z\left(\Psi_{\alpha, i}\right)=k$. Здесь $\operatorname{diam} Z\left(\Psi_{\alpha, i}\right)$ есть расстояние между крайней левой и крайней правой точками $Z\left(\Psi_{\alpha, i}\right)$. Тогда $l\left(D_{k}\right) \leqslant \operatorname{const} \rho^{k} \mathrm{c}$ теми же const и $\rho$, что и в $5^{\circ}$.

$7^{\circ}$. Собственные функции убывают экспоненциально вне их с.н. Мы сформулируем это свойство следуюшим образом: найдется $\gamma_{1}=\gamma_{1}(\varepsilon, \omega)>0$ такое, что для почти всех $\alpha$

$$
\begin{aligned}
\lim _{|m| \rightarrow \infty} \max _{\Psi_{\alpha+m \omega, i} \in \Phi_{\alpha+m \omega}} & \frac{1}{|m|} \ln \left|\Psi_{\alpha+m \omega, i}(m)\right| \\
& =\lim _{|m| \rightarrow \infty} \min _{\alpha+m \omega, i} \in \Phi_{\alpha+m \omega} \frac{1}{|m|} \ln \left|\Psi_{\alpha+m \omega, i}(m)\right| \leqslant-\gamma_{1} .
\end{aligned}
$$

Следуюшие свойства описьвают структуру $\Lambda_{\alpha}$ около точки, где $\Lambda_{\alpha}$ принимает максимальное значение.

$8^{\circ}$. Сушествует единственная точка $\alpha_{\max }$, в которой $\Lambda_{\alpha}$ однозначна, и при $\alpha \neq \alpha_{\max }$ справедливо неравенство $\lambda_{\alpha, i}<\Lambda_{\alpha_{\max }}$ для всех $\lambda_{\alpha, i} \in \Lambda_{\alpha}$. Многозначная функция $\Lambda_{\alpha}$ непрерывна в точке $\alpha_{\max }$ в том смысле, что при некотором $\sigma=\sigma(\omega, F)>0$ и $\Lambda_{\alpha_{\max }}=\Lambda_{\max }$

$$
\lim _{\alpha \rightarrow \alpha_{\max }} \max _{\lambda_{\alpha, i} \in \Lambda_{\alpha}} \frac{\Lambda_{\max }-\lambda_{\alpha, i}}{\left(\alpha-\alpha_{\max }\right)^{2}}=\lim _{\alpha \rightarrow \alpha_{\max }} \min _{\lambda_{\alpha, i} \in \Lambda_{\alpha}} \frac{\Lambda_{\max }-\lambda_{\alpha, i}}{\left(\alpha-\alpha_{\max }\right)^{2}}=\sigma .
$$

Последнее соотношение не означает, что в целой окрестности $\alpha_{\max }$ функция $\Lambda_{\alpha}$ непрерывна. Более того, точки разрьва $\Lambda_{\alpha}$ накапливаются к точке $\alpha_{\max }$. Однако, относительная мера тех $\alpha$, где функция $\Lambda_{\alpha}$ неоднозначна и не непрерьвна, убывает экспоненциально. Размеры разрывов убывают с еше большей скоростью. Нам понадобится это свойство в такой формулировке: если $U_{\xi}=\left\{\alpha|| \alpha-\alpha_{\max } \mid<\xi\right\}$ и $U_{\xi}^{(1)} \subset U_{\xi}$ есть множество тех $\alpha$, где $\Lambda_{\alpha}$ однозначна, т.е. $Z\left(\Psi_{\alpha, 1}\right)=0$ при таких $\alpha$, то при некоторой const $>0$

$$
\exp \left\{- \text { const } \cdot \exp \left\{-\frac{\text { const }}{\xi}\right\}\right\} \leqslant \frac{l\left(U_{\xi}^{(1)}\right)}{l\left(U_{\xi}\right)} \leqslant \exp \left\{\text { const } \cdot \exp \left\{-\frac{\text { const }}{\xi}\right\}\right\} .
$$

Здесь const зависит от $\omega$ и $\alpha$, но не от $\varepsilon$. Примерный вид $\Lambda_{\alpha}$ в окрестности $\alpha_{\max }$ представлен на рис. 3.

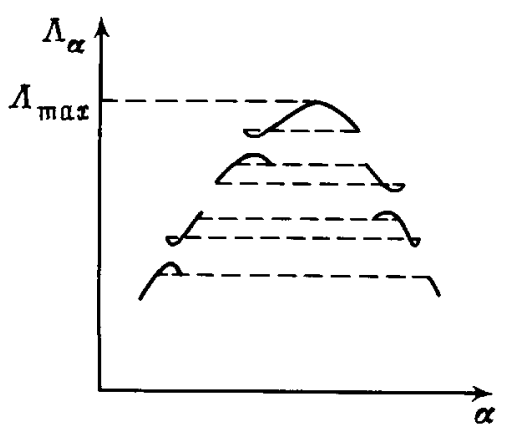

Рис. 3 
$9^{\circ}$. При доказательстве теоремы 1 мы будем пользоваться более сильным вариантом утверждения об экспоненциальном убывании с.ф. $\Psi_{\alpha, i} \in \Phi_{\alpha}$ для $\alpha$, близких к $\alpha_{\max }$. А именно, для некоторого $\gamma_{2}=\gamma_{2}(\varepsilon, \omega)>0$

$$
\lim _{\xi \rightarrow 0} \lim _{m \rightarrow \infty} \sup _{\alpha \in U^{(1)}} \frac{\ln \left|\Psi_{\alpha, 1}(m)\right|}{|m|}=\lim _{\xi \rightarrow 0} \lim _{m \rightarrow \infty} \inf _{\alpha \in U_{\xi}^{(1)}} \frac{\ln \left|\Psi_{\alpha, 1}(m)\right|}{|m|}=-\gamma_{2} .
$$

Выполнение всех свойств $1^{\circ}-9^{\circ}$ для типичных $\omega$ и $\alpha$ обсуждается в $\oint 4$ после описания доказательства ЛА.

\section{§ 2. Доказательство теоремы 1}

Положим $Z_{t}(\alpha, \varepsilon, x)=\sum_{b_{t}: b(t)=x} \pi\left(b_{t}\right)$. Тогда вся статистическая сумма $Z_{t}(\alpha, \varepsilon)=\sum_{x} Z_{t}(\alpha, \varepsilon, x)$ и

$$
r_{t}=\frac{\sum_{b_{t} \in \Omega_{t}} b^{2}(t) \pi\left(b_{t}\right)}{\sum_{b_{t} \in \Omega_{t}} \pi\left(b_{t}\right)}=\frac{\sum_{x} x^{2} Z_{t}(\alpha, \varepsilon, x)}{Z_{t}(\alpha, \varepsilon)}
$$

Для $Z_{t}(\alpha, \varepsilon, x)$ можем написать

$$
Z_{t}(\alpha, \varepsilon, x)=\varepsilon Z_{t-1}(\alpha, \varepsilon, x-1)+\varepsilon Z_{t-1}(\alpha, \varepsilon, x+1)+\exp \{F(x \omega+\alpha)\} Z_{t-1}(\alpha, \varepsilon, x) .
$$

Последнее соотношение позволяет представить статистическую сумму $Z_{t}(\alpha, \varepsilon)$ в виде

$$
Z_{t}(\alpha, \varepsilon)=\left(e, H_{\alpha}^{t} \delta_{0}\right)
$$

где $e=\{e(x)\}, e(x) \equiv 1, \delta_{0}(x)=1$ при $x=0$ и $\delta_{0}(x)=0$ при остальных $x$. Точно так же

$$
Z_{t}^{(0)}(\alpha, \varepsilon)=\sum x^{2} Z_{t}(\alpha, \varepsilon, x)=\left(f, H_{\alpha}^{t} \delta_{0}\right)
$$

где $f(x)=x^{2}$.

Допустим, что $\omega$ и $\alpha$ таковы, что при достаточно малых $\varepsilon$ имеет место локализация Андерсона со всеми свойствами, перечисленными в $\S 1$. Мы можем написать

$$
\begin{aligned}
Z_{t}(\alpha, \varepsilon) & =\sum_{i} \lambda_{\alpha, i}^{t} \Psi_{\alpha, i}(0) \sum_{x} \Psi_{\alpha, i}(x) \\
Z_{t}^{(0)}(\alpha, \varepsilon) & =\sum_{i} \lambda_{\alpha, i}^{t} \Psi_{\alpha, i}(0) \sum_{x} x^{2} \Psi_{\alpha, i}(x) .
\end{aligned}
$$

Начиная с этого момента, мы обсуждаем только $Z_{t}(\alpha, \varepsilon)$ и в конце параграффа указываем необходимые изменения для $Z_{t}^{(0)}(\alpha, \varepsilon)$. С помошюю $3^{\circ}, 4^{\circ}$ из $\S 1$ получаем

$$
\text { (8) } \begin{aligned}
Z_{t}(\alpha, \varepsilon) & =\sum_{-\infty<m<\infty i: \operatorname{pos}\left(\Psi_{\alpha, i}\right)=m} \lambda_{\alpha, i}^{t} \Psi_{\alpha, i}(0) \sum_{x} \Psi_{\alpha, i}(x) \\
& =\sum_{-\infty<m<\infty} \sum_{\alpha+m \omega, i \in \Phi_{\alpha+m \omega}} \lambda_{\alpha+m \omega, i}^{t} \Psi_{\alpha+m \omega, i}(-m) \sum_{x} \Psi_{\alpha+m \omega, i}(x) .
\end{aligned}
$$


В последнем выражении индекс $i$ нумерует различные с.ф., принадлежащие $\Phi_{\alpha+m \omega}$. Мы покажем, что в типичной ситуации основной вклад в (8) вносит единственное слагаемое при определенных значениях $m$ и $i$.

Разложим сумму (8) на несколько частей. Возьмем интервал $-t^{1 / 3} \leqslant m \leqslant t^{1 / 3}$, выберем $C_{1}(\delta)=C_{1}$ и рассмотрим окрестность $U^{(t)}=\left\{\alpha:\left|\alpha-\alpha_{\max }\right| \leqslant C_{1} t^{-1 / 3}\right\}$. Точное значение $C_{1}$ определим позже. При заданных $\omega$ и $\alpha$ найдем такие $m_{j}$, $\left|m_{j}\right| \leqslant C_{1} t^{1 / 3}$, что $\alpha+m_{j} \omega \in U^{(t)}, j=1, \ldots, J$. Пусть $\omega$ и $\alpha$ таковы, что $J \leqslant J_{0}(\delta)$, где $J_{0}(\delta)$, не зависяшее от $t$, также будет определено позже. Обозначим $x_{j}=\left(\alpha+m_{j} \omega-\alpha_{\max }\right) t^{1 / 3}, y_{j}=m_{j} t^{-1 / 3}$. Положим

$$
Z_{t}^{(1)}(\alpha, \varepsilon)=\sum_{\left|m_{j}\right| \leqslant t^{1 / 3} \Psi_{\alpha+m_{j} \omega, i} \in \Phi_{\alpha+m_{j} \omega}} \lambda_{\alpha+m_{j} \omega, i}^{t} \Psi_{\alpha+m_{j} \omega, i}\left(-m_{j}\right) \sum_{x} \Psi_{\alpha+m_{j} \omega, i}(x),
$$

$$
Z_{t}^{(2)}(\alpha, \varepsilon)=\sum_{j=1}^{J} \sum_{\Psi_{\alpha+m_{j} \omega, i} \in \Phi_{\alpha+m_{j} \omega}} \lambda_{\alpha+m_{j} \omega, i}^{t} \Psi_{\alpha+m_{j} \omega, i}\left(-m_{j}\right) \sum_{x} \Psi_{\alpha+m_{j} \omega, i}(x),
$$

$$
Z_{t}^{(3)}(\alpha, \varepsilon)=\sum_{|m|>C_{1} t^{1 / 3}} \sum_{\Psi_{\alpha+m \omega, i} \in \Phi_{\alpha+m \omega, i}} \lambda_{\alpha+m \omega, i}^{t} \Psi_{\alpha+m \omega, i}(-m) \sum_{x} \Psi_{\alpha+m \omega, i}(x),
$$

$$
Z_{t}^{(4)}(\alpha, \varepsilon)=\sum_{\substack{|m| \leqslant C_{1} t^{1 / 3} \\ m \neq m_{j}}} \sum_{\Psi_{\alpha+m \omega, i} \in \Phi_{\alpha+m \omega, i}} \lambda_{\alpha+m \omega, i}^{t} \Psi_{\alpha+m \omega, i}(-m) \sum_{x} \Psi_{\alpha+m \omega, i}(x) .
$$

Мы покажем, что при некоторых естественных предположениях

$$
Z_{t}^{(2)}(\alpha, \varepsilon) \geqslant Z_{t}^{(1)}(\alpha, \varepsilon) \gg Z_{t}^{(3)}(\alpha, \varepsilon)+Z_{t}^{(4)}(\alpha, \varepsilon) .
$$

Мы формулируем эти предположения в виде требований к $\omega$ и $\alpha$, которые будут обсуждаться в конце этого параграфа и в $\S 4$.

$\left(\mathrm{H}_{1}\right)$ Пусть $\omega$ и $\alpha$ таковы, что для всех $\alpha+m_{j} \omega, j=1,2, \ldots, J \leqslant J_{0}$ множество $\Phi_{\alpha+m_{j} \omega}$ состоит из единственной с.ф.. Тогда $i$ в $(9),(10)$ принимает единственное значение $i=1$.

Используя $8^{\circ}$ из $§ 1$, можем написать

$$
\begin{aligned}
\lambda_{\alpha+m_{j} \omega, 1}^{t} & =\Lambda_{\max }^{t}\left(\frac{\lambda_{\alpha+m_{j} \omega, 1}}{\Lambda_{\max }}\right)^{t}=\Lambda_{\max }^{t}\left(1-\frac{\Lambda_{\max }-\lambda_{\alpha+m_{j} \omega, 1}}{\Lambda_{\max }}\right)^{t} \\
& =\Lambda_{\max }^{t}\left(1-\frac{\Lambda_{\max }-\Lambda_{\alpha+m_{j} \omega}}{\Lambda_{\alpha_{\max }}}\right)^{t}=\Lambda_{\max }^{t}\left(1-\frac{\sigma x_{j}^{2}(1+o(1))}{t^{2 / 3}}\right)^{t} \\
& =\Lambda_{\max }^{t} \exp \left\{-t^{1 / 3} \sigma x_{j}^{2}(1+o(1))\right\} .
\end{aligned}
$$

С помощью $9^{\circ}$ из $§ 1$ получаем

$$
\Psi_{\alpha+m_{j} \omega, 1}\left(-m_{j}\right)=\exp \left\{-\gamma_{2}\left|m_{j}\right|(1+o(1))\right\}=\exp \left\{-\gamma_{2} t^{1 / 3}|y|(1+o(1))\right\}
$$


Таким образом,

$$
\lambda_{\alpha+m_{j} \omega, 1}^{t} \Psi_{\alpha+m_{j} \omega, 1}\left(-m_{j}\right)=\Lambda_{\max }^{t} \exp \left\{-t^{1 / 3}\left(\sigma x_{j}^{2}+\gamma_{2}\left|y_{j}\right|\right)(1+o(1))\right\}
$$

Последнее соотношение показывает, что для слагаемых, входяших в $Z_{t}^{(1)}(\alpha, \varepsilon)$ и $Z_{t}^{(2)}(\alpha, \varepsilon), \lambda_{\alpha+m_{j} \omega, 1}^{t} \Lambda_{\alpha \max }^{-t}$ и $\Psi_{\alpha, i}\left(-m_{j}\right)$ имеют одинаковый порядок. Для слагаемых, входяших в $Z_{t}^{(3)}(\alpha, \varepsilon)$ и $Z_{t}^{(4)}(\alpha, \varepsilon)$, один из этих сомножителей становится сушественно менњше. Этим, грубо говоря, объясняется то, что основной вклад в статистическую сумму $Z_{t}(\alpha, \varepsilon)$ вносит конечная сумма $Z_{t}^{(2)}(\alpha, \varepsilon)$.

$\left(\mathrm{H}_{2}\right)$ Среди всех чисел $\sigma x_{j}^{2}+\gamma_{2}\left|y_{j}\right|,\left|y_{j}\right| \leqslant 1$, имеется только одно число с $j=j_{1}$, для которого

$$
\sigma x_{j_{1}}^{2}+\gamma_{2}\left|y_{j_{1}}\right|+\beta_{1}<\sigma x_{j}^{2}+\gamma_{2}\left|y_{j}\right|, \quad j \neq j_{1} .
$$

Среди всех чисел $\sigma x_{j}^{2}+\gamma_{2}\left|y_{j}\right|,\left|y_{j}\right| \leqslant C_{1}(\delta)$, имеется только одно число с $j=j_{0}$, для которого

$$
\sigma x_{j_{0}}^{2}+\gamma_{2}\left|y_{j_{0}}\right|+\beta_{1}<\sigma x_{j}^{2}+\gamma_{2}\left|y_{j}\right|, \quad j \neq j_{0} .
$$

Здесь $\beta_{1}$ - еще одна постоянная, не зависящая от $t$ и зависящая только от $\omega, \alpha, \varepsilon$ и $\delta$.

Если выполнено $\left(\mathrm{H}_{2}\right)$, то основной вкладв $Z_{t}^{(1)}(\alpha, \varepsilon)$ вносит единственное слагаемое с $j=j_{0}$. В принципе возможно, что $j_{1}=j_{0}$. Если же $j_{1} \neq j_{0}$, то $Z_{t}^{(2)}(\alpha, \varepsilon) \gg$ $Z_{t}^{(1)}(\alpha, \varepsilon)$.

Покажем теперь, что $Z_{t}^{(3)}(\alpha, \varepsilon)$ мало по сравнению с $Z_{t}^{(1)}(\alpha, \varepsilon)$, если $C_{1}$ выбрано достаточно большим. Мы имеем

$$
\left|Z_{t}^{(3)}(\alpha, \varepsilon)\right| \leqslant \Lambda_{\max }^{t} \sum_{|m|>C_{1} t^{1 / 3}} \sum_{\Psi_{\alpha+m \omega, i} \in \Phi_{\alpha+m \omega}}\left|\Psi_{\alpha+m \omega, i}(-m)\right| \sum_{x}\left|\Psi_{\alpha+m \omega, i}(x)\right| .
$$

$\left(\mathrm{H}_{3}\right)$ При некоторой постоянной $C_{2}=C_{2}(\varepsilon, \delta)$ числа $\omega$ и $\alpha$ таковы, что при всех допустимых $i$

$$
\left|\sum_{x} \Psi_{\alpha+m \omega, i}(x)\right| \leqslant C_{2} m^{2}
$$

и $\operatorname{diam} Z\left(\Psi_{\alpha+m \omega, i}\right) \leqslant \ln ^{2} m+C_{2}$. На самом деле в правой части (13) можно поставить гораздо более медленно меняющуюся функцию, но для наших целей достаточно $\mathrm{m}^{2}$.

Из (4) имеем при всех $m,|m| \geqslant C_{1} t^{1 / 3}$, и $i$

$$
\left|\Psi_{\alpha+m \omega, i}(-m)\right| \leqslant \exp \left\{-\frac{\gamma_{1}}{2}|m|\right\}
$$

Поэтому

$$
\left|Z_{t}^{(3)}(\alpha, \varepsilon)\right| \leqslant \Lambda_{\max }^{t} \exp \left\{-\frac{\gamma_{1}}{2} C_{1} t^{1 / 3}\right\} \cdot \text { const } .
$$

Если $\omega$ и $\alpha$ таковы, что

$$
\frac{\gamma_{1} C_{1}}{2} \geqslant \sigma x_{j_{1}}^{2}+\gamma_{2}\left|y_{j_{1}}\right|+\beta_{1}
$$


TO

$$
\left|Z_{t}^{(3)}(\alpha, \varepsilon)\right| \leqslant \text { const } \cdot \exp \left\{-\beta_{1} t^{1 / 3}\right\} Z_{t}^{(1)}(\alpha, \varepsilon) \leqslant \text { const } \cdot \exp \left\{-\beta_{1} t^{1 / 3}\right\} Z_{t}^{(2)}(\alpha, \varepsilon),
$$

что и требовалось показать.

Покажем теперь, что $Z_{t}^{(4)}(\alpha, \varepsilon)$ мало по сравнению с $Z_{t}^{(1)}(\alpha, \varepsilon)$ в силу малости всех $\lambda_{\alpha+m \omega, i}^{t}$. Мы оценим часть $Z_{t}^{(4)}(\alpha, \varepsilon)$, отвечаюшую $m \geqslant 0$. Другая часть оценивается аналогично. Имеем

$$
Z_{t}^{(4,+)}(\alpha, \varepsilon)=\sum_{\substack{0 \leqslant m \leqslant C_{1} t^{1 / 3} \\ \operatorname{dist}\left(\alpha+m \omega, \alpha_{\max }\right) \geqslant \frac{C_{1}}{t^{1 / 3}}}} \sum_{\Psi_{\alpha+m \omega, i} \in \Phi_{\alpha+m \omega}} \lambda_{\alpha+m \omega, i}^{t} \cdot \Psi_{\alpha+m \omega, i}(-m) \sum_{x} \Psi_{\alpha+m \omega, i}(x) .
$$

Поскольку $\alpha+m \omega$ далеко от $\alpha_{\max }$, мы воспользуемся оценкой

$$
\lambda_{\alpha+m \omega, i} \leqslant \Lambda_{\max }\left(1-\frac{\sigma C_{1}^{2}}{t^{1 / 3}}(1+o(1))\right) .
$$

Используя очевидное неравенство $\left|\Psi_{\alpha+m \omega, i}\right| \leqslant 1$ и (13), мы можем написать для всех достаточно больших $t$

$$
\left|Z_{t}^{(4,+)}(\alpha, \varepsilon)\right| \leqslant \text { const } t \ln ^{2} t \Lambda_{\max }^{t} \exp \left\{-\frac{\sigma}{2} C_{1}^{2} t^{1 / 3}\right\} .
$$

Оценим теперь $Z_{t}^{(1)}(\alpha, \varepsilon)$ снизу. Как следует из $\left(\mathrm{H}_{2}\right)$, основной вклад в $Z_{t}^{(1)}(\alpha, \varepsilon)$ вносит слагаемое с $j=j_{1}$. Поэтому достаточно рассмотреть

$$
\lambda_{\alpha+m_{j_{1}} \omega, 1}^{t} \cdot \Psi_{\alpha+m_{j_{1}} \omega, 1}\left(-m_{j_{1}}\right) \sum_{x} \Psi_{\alpha+m_{j_{1}} \omega, 1}(x) .
$$

Начнем со следующей леммы.

Лемма 1. Для любого $\delta>0$ можно найти такое $C_{3}=C_{3}(\delta)>0$, что

$$
l\left\{(\omega, \alpha):\left|\alpha+m \omega-\alpha_{\max }\right| \geqslant C_{3} t^{1 / 3} \text { nри всех } 0 \leqslant m \leqslant t^{1 / 3}\right\} \geqslant 1-\frac{1}{4} \delta^{2} .
$$

Утверждение леммы очевидно, и доказательство опускается. Пусть $O_{t}$ есть множество тех $\omega$, для которых условная мера $\alpha$, удовлетворяюших предыдущему неравенству, превьшшает $1-\delta / 2$. Тогда $l\left(O_{t}\right) \geqslant 1-\delta / 2$.

Для $\left|\Psi_{\alpha+m_{j_{1}} \omega, 1}\left(-m_{j_{1}}\right)\right|$ мы используем оценку (см. $\left.9^{\circ}, \S 1\right)$

$$
\left|\Psi_{\alpha+m_{j_{1}} \omega, 1}\left(-m_{j_{1}}\right)\right| \geqslant \exp \left\{-\gamma_{2} m_{j_{1}}(1+o(1))\right\}
$$

и $\left|\sum_{x} \Psi_{\alpha+m_{j_{1}} \omega, 1}(x)\right| \leqslant 1+$ const $\varepsilon \varepsilon$. Последняя оценка, где const зависит от $\omega$ и $\alpha$, легко вытекает из доказательства ЛА (см. §3). Для $\omega \in O_{t}$ можем теперь написать

$$
\begin{aligned}
Z_{t}^{(1)}(\alpha, \varepsilon) & \geqslant \Lambda_{\max }^{t}\left(1-\sigma \frac{C_{3}^{2}}{t^{2 / 3}}(1+o(1))\right)^{t} \exp \left\{-\gamma_{2} t^{1 / 3}(1+o(1))\right\} \\
& =\Lambda_{\max }^{t} \exp \left\{-t^{1 / 3}\left(\sigma C_{3}^{2}+\gamma_{2}\right)(1+o(1))\right\} .
\end{aligned}
$$


При заданном $C_{3}$, выбрав $C_{1}$ достаточно большим, а $\beta_{1}$ достаточно малым, можем написать

$$
\frac{Z_{t}^{(4,+)}(\alpha, \varepsilon)}{Z_{t}^{(1)}(\alpha, \varepsilon)} \leqslant e^{-\beta_{1} t} .
$$

Обсуждение условий $\left(\mathrm{H}_{1}\right)-\left(\mathrm{H}_{3}\right)$. Возьмем произвольное $C_{1}>1$. Можно найти такое $J_{0}=J_{0}\left(C_{1}, \delta\right)$, что мера тех пар $(\omega, \alpha)$, где $J \leqslant J_{0}$, превосходит $1-\frac{1}{64} \delta^{2}$. Разумеется, $J_{0} \rightarrow \infty$ при $C_{1} \rightarrow \infty$, но это для нас несушественно, поскольку вначале $t \rightarrow \infty$.

При заданном $C_{1}$ условная мера тех $\alpha \in U^{(t)}$, где $\Phi_{\alpha}$ многозначна или принимает пустое множество значений, не превосходит

$$
\text { const } \cdot \exp \left\{- \text { const } \cdot C_{1} t^{1 / 3}\right\} \text {. }
$$

Это следует из оценки размеров резонансных зон (см. $33, \S 4)$. Здесь const зависят только от $\omega$. Выбирая те $\alpha$, для которых $\alpha+m \omega$ не попадает в это множество при $|m| \leqslant C_{1} t^{1 / 3}$, мы получаем $\left(\mathrm{H}_{1}\right)$.

Обратимся к неравенству (14). В нем $\gamma_{1}, x_{j_{1}}, y_{j_{1}}$ представляют собой функции $\omega$ и $\alpha$, а также $\varepsilon$ и $F$, что для нас несушественно. Поэтому мы можем выбрать $C_{1}$ настолько большим, что вероятность пар $(\omega, \alpha)$, для которых справедливо $(14)$, превосходит $1-\frac{1}{64} \delta^{2}$.

Для проверки $\left(\mathrm{H}_{2}\right)$ будем считать $\omega$ фиксированным и менять значение $\alpha$. Величина $\gamma_{2}$ зависит только от $\omega$ и $\varepsilon$, но не от $\alpha$. При заданных $m_{j^{\prime}}, m_{j^{\prime \prime}},\left|m_{j^{\prime}}\right| \leqslant C_{1} t^{1 / 3}$, $\left|m_{j^{\prime \prime}}\right| \leqslant C_{1} t^{1 / 3}$, рассмотрим множество тех $\alpha$, для которых $\left|\alpha+m_{j^{\prime}} \omega-\alpha_{\max }\right| \leqslant$ $C_{1} t^{-1 / 3}$ и $\left|\alpha+m_{j^{\prime \prime}} \omega-\alpha_{\max }\right| \leqslant C_{1} t^{-1 / 3}$. Оно распадается на отдельные отрезки. Соотношение $\sigma x_{j^{\prime}}^{2}+\gamma_{2}\left|y_{j^{\prime}}\right|=\sigma x_{j^{\prime \prime}}^{2}+\gamma_{2}\left|y_{j^{\prime \prime}}\right|$ можно рассматривать как уравнение на $\alpha$, имеюшее в каждом таком отрезке не более одного решения, поскольку это уравнение линейное. Возьмем $\alpha$ вне окрестностей этих решений размера $\beta_{3} t^{-1 / 3}$, где $\beta_{3}=\beta_{3}(\omega, \varepsilon)$ достаточно мало, так, чтобы мера дополнения к этим окрестностям была больше, чем $1-\delta / 8$. Этим выбором определяется значение $\beta_{3}=\beta_{3}(\omega, \varepsilon)>0$.

Условия $\left(\mathrm{H}_{3}\right)$ вытекает из свойств ЛА. Мы обсуждаем соответствующие вопросы в $\oint 3, \S 4$.

Из $\left(\mathrm{H}_{1}\right)-\left(\mathrm{H}_{3}\right)$ вытекает, что основной вклад в $Z_{t}(\alpha, \varepsilon)$ вносит слагаемое

$$
\lambda_{\alpha+m_{j_{0}} \omega, 1}^{t} \cdot \Psi_{\alpha+m_{j_{0}} \omega, 1}\left(-m_{j_{0}}\right) \sum_{x} \Psi_{\alpha+m_{j_{0}} \omega, 1}(x) .
$$

Точно так же можно показать, что вероятности $Z_{t}(\alpha, \varepsilon, x) / Z_{t}(\alpha, \varepsilon)$ убывают экспоненциально как функции $x-m_{j_{0}}$.

Рассуждения для оценки $Z_{t}^{(0)}(\alpha, \varepsilon)$ в основном такие же. Они показывают, что и здесь основной вклад вносит то же слагаемое

$$
\begin{aligned}
\lambda_{\alpha+m_{j_{0}} \omega, 1}^{t} \cdot \Psi_{\alpha+m_{j_{0}} \omega, 1}\left(-m_{j_{0}}\right) \sum_{x}\left(x+m_{j_{0}}\right)^{2} \Psi_{\alpha+m_{j_{0} \omega, 1}}(x) \\
=m_{j_{0}}^{2} \lambda_{\alpha+m_{j_{0}} \omega, 1}^{t} \cdot \Psi_{\alpha+m_{j_{0} \omega, 1}}\left(-m_{j_{0}}\right) \sum_{x} \Psi_{\alpha+m_{j_{0} \omega, 1}}(x)+\cdots,
\end{aligned}
$$

где многоточием отмечены члены, малые по сравнению с выписанными слагаемыми. 


\section{§ 3. Локализация Андерсона оператора $H_{\alpha}$}

В этом параграфе мы приводим подробное описание доказательство ЛА оператора $H_{\alpha}$, следуя, в основном, работе [4]. Подход этой работы отличается от других подходов, в частности, в [2] и [3], тем, что в [4] разработана техника построения локализованных с.ф. и с.н. В определенном смысле подход [4] ближе к теории КАМ, чем [2], [3]. Хотя этот параграф̆ и не содержит полного доказательства ЛА, мы надеемся, что заинтересованный читатель сможет восстановить недостаюшие детали.

Выпишем оператор $H_{\alpha}$ в чуть более обшем виде:

$$
\left(H_{\alpha} \Psi\right)(x)=\varepsilon \Psi(x+1)+\varepsilon \Psi(x-1)+V(x \omega+\alpha) \Psi(x) .
$$

Здесь $V$ - периодическая $C^{2}$-функция Морса, имеюшая две невырожденные критические точки. При этом сушественно, что $V$ принимает каждое значение не более чем в двух точках. Случай более общих $V$ рассмотрен в [3] и [5].

Как уже отмечалось раньше, ЛА означает, что оператор $H_{\alpha}$ имеет чисто точечньй спектр с локализованньми с.ф. Если $\left\{\lambda_{\alpha, i}\right\}$ - набор с.з. $H_{\alpha}$, то $\left\{\lambda_{\alpha, i}\right\}=\left\{\lambda_{\alpha+\omega, i}\right\}$. Иными словами, спектр $H_{\alpha}$, понимаемый как множество его собственных значений, инвариантен относительно врашения $T_{\omega} \alpha=\alpha+\omega(\bmod 1)$. В случае иррациональных $\omega$ не существует измеримых инвариантных функций, кроме констант, что означает, что спектр $\left\{\lambda_{\alpha, i}\right\}$ не является измеримой функцией. Наш подход показьвает, как справиться с этой неизмеримостью, т.е. как ввести своеобразную униформацию спектра. А именно, мы строим “измеримую функцию” $\Lambda_{\alpha}$ таким образом, что весь спектр $\left\{\lambda_{\alpha, i}\right\}=\bigcup_{-\infty<m<\infty} \Lambda_{\alpha+m \omega}$. Кавычки поставлены потому, что $\Lambda_{\alpha}$ является, вообще говоря, многозначной функцией. Как уже отмечалось, последнее соотношение надо трактовать таким образом, что набор всех с.з. $H_{\alpha}$ состоит из значений $\Lambda_{\alpha}$ вдоль траектории поворота $T_{\omega}$.

Мы уже упоминали о том, что в основе построения $\Lambda_{\alpha}$ и соответствующей $\Phi_{\alpha}$ лежит идея о том, что каждая с.ф. $\Psi_{\alpha, i}$ имеет с.н. $Z\left(\Psi_{\alpha, i}\right)$, в окрестности которого она главным образом сосредоточена. Естественное требование к с.н. состоит в том, что если $Z\left(\Psi_{\alpha, i}\right)$ есть с.н. $\Psi_{\alpha, i}$ и $\Psi_{\alpha+\omega, i}(x)=\Psi_{\alpha, i_{1}}(x+1)$, то $Z\left(\Psi_{\alpha+\omega, i}\right)=Z\left(\Psi_{\alpha, i}\right)-1$.

Пусть, как и выше, $\operatorname{pos}\left(\Psi_{\alpha, i}\right)$ есть крайняя левая точка $Z\left(\Psi_{\alpha, i}\right)$. Положим $\Phi_{\alpha}=$ $\left\{\Psi_{\alpha, i} \mid \operatorname{pos}\left(\Psi_{\alpha, i}\right)=0\right\}, \Lambda_{\alpha}=\left\{\lambda_{\alpha, i} \mid \Psi_{\alpha, i} \in \Phi_{\alpha}\right\}$. Если $S$ есть сдвиг влево, $(S \Psi)(x)=$ $\Psi(x+1)$, то

$$
\left\{\Psi_{\alpha, i}\right\}=\bigcup_{-\infty<m<\infty} S^{m} \Phi_{\alpha+m \omega}, \quad\left\{\lambda_{\alpha, i}\right\}=\bigcup_{-\infty<m<\infty} \Lambda_{\alpha+m \omega} .
$$

Построение $\Psi_{\alpha, i}, Z\left(\Psi_{\alpha, i}\right), \lambda_{\alpha, i}$ проводится по индукции и основано на теории возмущений. На каждом шагу индукции мы предполагаем, что мы имеем приближенные с.ф. $\Psi_{\alpha, i}^{(n)}$ и их с.н. $Z\left(\Psi_{\alpha, i}^{(n)}\right)$ с $\operatorname{pos}\left(\Psi_{\alpha, i}^{(n)}\right)=0$, функцию $\Phi_{\alpha}^{(n)}=\left\{\Psi_{\alpha, i}^{(n)}\right\}$ и приближенные с.з. $\Lambda_{\alpha}^{(n)}=\left\{\lambda_{\alpha, i}^{(n)} \mid \Psi_{\alpha, i}^{(n)} \in \Phi_{\alpha}^{(n)}\right\}$. После этого мы рассматриваем приближенньй базис

$$
\left\{S^{m} \Psi_{\alpha+m \omega, i}^{(n)},-\infty<m<\infty\right\}, \quad Z\left(S^{m} \Psi_{\alpha+m \omega, i}^{(n)}\right)=Z\left(\Psi_{\alpha+m \omega, i}^{(n)}\right)-m,
$$

и приближенньй спектр $\bigcup_{-\infty<m<\infty} \Lambda_{\alpha+m \omega}^{(n)}$. Функции $S^{m} \Psi_{\alpha+m \omega, i}^{(n)}$ неортогональны, но нормированы. Мы обсудим их приближение к ортогональности позже. 
Теперь мы остановимся на основном механизме убывания с.ф. вне их существенных носителей. Допустим, что ЛА уже установлена, и для данного $H_{\alpha}$ мы имеем отвечающие ему нормированные точные с.ф. $\Psi_{\alpha, i}$, их с.н. $Z\left(\Psi_{\alpha, i}\right)$, с.з. $\lambda_{\alpha, i}$ и, дополнительно, $\lambda_{\alpha, i_{1}} \neq \lambda_{\alpha, i_{2}}$ при $i_{1} \neq i_{2}$, т.е. кратность каждого с.з. равна 1 . Последнее свойство характерно для ЛА при типичных $\alpha$.

Возьмем какую-либо точную с.ф. $\Psi_{\alpha, i}$, выберем $m_{0}$ и рассмотрим функцию $\varphi_{\alpha, i}$, для которой $\varphi_{\alpha, i}(m)=\Psi_{\alpha, i}$ при $m \leqslant m_{0}$ и $\varphi_{\alpha, i}(m)=0$ при $m>m_{0}$. Легко проверить, что

$$
\begin{gathered}
H_{\alpha} \varphi_{\alpha, i}(m)=\lambda_{\alpha, i} \varphi_{\alpha, i}(m) \text { при } m<m_{0} \text { или } m>m_{0}+1, \\
H_{\alpha} \varphi_{\alpha, i}\left(m_{0}\right)=\lambda_{\alpha, i} \varphi_{\alpha, i}\left(m_{0}\right)-\varepsilon \Psi_{\alpha, i}\left(m_{0}+1\right), \\
\left(H_{\alpha} \varphi_{\alpha, i}\right)\left(m_{0}+1\right)=\lambda_{\alpha, i} \varphi_{\alpha, i}\left(m_{0}+1\right)+\varepsilon \Psi_{\alpha, i}\left(m_{0}\right) .
\end{gathered}
$$

Поэтому вектор $\left(H_{\alpha}-\lambda_{\alpha, i}\right) \varphi_{\alpha, i}=\chi$ имеет только две ненулевых компоненты $\chi\left(m_{0}\right)=$ $-\varepsilon \Psi_{\alpha, i}\left(m_{0}+1\right), \chi\left(m_{0}+1\right)=\varepsilon \Psi_{\alpha, i}\left(m_{0}\right)$ и, тем самым, ортогонален к $\Psi_{\alpha, i}$. Покажем, что

$$
\varphi_{\alpha, i}+\sum_{j \neq i} \frac{\left(\chi, \Psi_{\alpha, j}\right)}{\lambda_{\alpha, i}-\lambda_{\alpha, j}} \Psi_{\alpha, j}
$$

есть с.ф. $H_{\alpha}$ с с.з. $\lambda_{\alpha, i}$, в предположении, что последняя сумма сходится в $L^{2}$. Малые знаменатели, которые здесь присутствуют, напоминают малые знаменатели КАМ-теории. В нашей ситуации сходимость имеет место, так как "мальм знаменателям" отвечают достаточно "малые числители". Далее, $\chi=\sum_{j \neq i}\left(\chi, \Psi_{\alpha, j}\right) \Psi_{\alpha, j}$ и

$$
\begin{aligned}
H_{\alpha}\left(\varphi_{\alpha, i}+\sum_{j \neq i} \frac{\left(\chi, \Psi_{\alpha, j}\right)}{\lambda_{\alpha, i}-\lambda_{\alpha, j}} \Psi_{\alpha, j}\right) & =\lambda_{\alpha, i} \varphi_{\alpha, i}+\chi+\sum_{j \neq i} \frac{\lambda_{\alpha, j}\left(\chi, \Psi_{\alpha, j}\right)}{\lambda_{\alpha, i}-\lambda_{\alpha, j}} \Psi_{\alpha, j} \\
& =\lambda_{\alpha, i}\left(\varphi_{\alpha, i}+\sum_{j \neq i} \frac{\left(\chi, \Psi_{\alpha, j}\right)}{\lambda_{\alpha, i}-\lambda_{\alpha, j}}\right) .
\end{aligned}
$$

Поэтому при некоторой постоянной $A_{\alpha, i, m}$

$$
A_{\alpha, i, m_{\circ}} \Psi_{\alpha, i}=\varphi_{\alpha, i}+\sum_{j \neq i} \frac{\left(\chi, \Psi_{\alpha, j}\right)}{\lambda_{\alpha, i}-\lambda_{\alpha, j}} \Psi_{\alpha, j}
$$

Мы будем использовать последние соотношения в ситуациях, когда с.н. $\Psi_{\alpha, i}$ лежит слева от $m_{\circ}$. Тогда $A_{\alpha, i, m}$ экспоненциально близка к 1 как функция от расстояния $\operatorname{dist}\left(m_{\circ}, Z\left(\Psi_{\alpha, i}\right)\right)$. Напишем (16) при $m=m_{\circ}+1$ :

$$
\begin{aligned}
A_{\alpha, i, m_{\circ}} \Psi_{\alpha, i}\left(m_{\circ}+1\right)= & \varepsilon \Psi_{\alpha, i}\left(m_{\circ}+1\right) \sum_{j \neq i} \frac{\Psi_{\alpha, j}\left(m_{\circ}\right) \Psi_{\alpha, j}\left(m_{\circ}+1\right)}{\lambda_{\alpha, i}-\lambda_{\alpha, j}} \\
& +\varepsilon \Psi_{\alpha, i}\left(m_{\circ}\right) \sum_{j \neq i} \frac{\Psi_{\alpha, j}^{2}\left(m_{\circ}+1\right)}{\lambda_{\alpha, i}-\lambda_{\alpha, j}} .
\end{aligned}
$$


Введем усеченную резольвенту

$$
\Gamma_{i}\left(\lambda, m_{1}, m_{2}\right)=\sum_{j \neq i} \frac{\Psi_{\alpha, j}\left(m_{1}\right) \Psi_{\alpha, j}\left(m_{2}\right)}{\lambda-\lambda_{\alpha, j}} .
$$

Из (17) вытекает

$$
\frac{\Psi_{\alpha, i}\left(m_{\circ}+1\right)}{\Psi_{\alpha, i}\left(m_{\circ}\right)}=\varepsilon \frac{\Gamma_{i}\left(\lambda_{\alpha, i} ; m_{\circ}, m_{\circ}+1\right)}{A_{\alpha, i, m_{\circ}}+\varepsilon \Gamma_{i}\left(\lambda_{\alpha, i} ; m_{\circ}+1, m_{\circ}+1\right)} .
$$

Поскольку коэффициенты $A_{\alpha, i, m}$ экспоненциально стремятся к 1 с ростом $m_{\circ}$, основной вклад в суммы (18), участвуюшие в (19), дают слагаемые, отвечаюшие с.ф., сосредоточенным около $m_{\circ}$, так как для таких $j$ значения $\Psi_{\alpha, j}(m)$ порядка 1 , а для остальных $j$ они убывают по мере удаления с.н. от $m_{\circ}$. Как уже упоминалось, основная тактика при доказательстве ЛА состоит в том, чтобы сделать малые знаменатели $\lambda_{\alpha, i}-\lambda_{\alpha, j}$ не слишком малыми, а соответствуюшие им числители не слишком большими. Тогда ряды (18) будут достаточно быстро сходиться и отношение

$$
\varepsilon \frac{\Gamma_{i}\left(\lambda_{\alpha, i} ; m_{\circ}+1, m_{\circ}+1\right)}{1+\varepsilon \Gamma_{i}\left(\lambda_{\alpha, i} ; m_{\circ}, m_{\circ}+1\right)}=\Theta\left(\lambda_{\alpha, i} ; \alpha+m_{\circ} \omega\right)
$$

можно рассматривать как более или менее регулярную функцию $\alpha+m_{\circ} \omega$. Мы можем теперь написать

$$
\frac{\Psi_{\alpha, i}\left(m_{\circ}+k\right)}{\Psi_{\alpha, i}\left(m_{\circ}\right)}=\varepsilon^{k} \prod_{r=m_{\circ}}^{m_{\circ}+k-1} \Theta\left(\lambda_{\alpha, i} ; \alpha+r \omega\right) \cdot R_{m_{\circ}}^{m_{\circ}+k-1},
$$

где $R_{m_{\circ}}^{m_{\circ}+k-1}$ - остаточньй член порядка 1 , связанньй с тем, что мы заменили все $A_{\alpha, i, m_{\circ}}$ на 1. Произведение в (20) может быть оценено с помощью эргодической теоремы, причем $\lambda_{\alpha, i}$ можно считать параметром. Мы видим также. что интеграл от логарифма модуля $\Theta\left(\lambda_{\alpha, i} ; \alpha\right)$ определяет показатель Ляпунова, отвечающий с.ф. $\Psi_{\alpha, i}$. Отношение в левой части (20) экспоненщиально стремится к нулю, если $\varepsilon$ достаточно мало.

Аналогичные формулы могут быть выписаны, когда $m_{\circ}$ лежит слева от с.н. $\Psi_{\alpha, i}$. В таком случае надо брать $\varphi$, равные нулю при $m<m_{\circ}$.

В основной индуктивной процедуре мы используем аналогичные формулы, в которых вместо точных с.ф. фигурируют приближенные с.ф.

Теперь мы проделаем явно два первых шага основной индуктивной процедуры. На нулевом шаге полагаем

$$
\Psi_{\alpha, i}^{(0)}=\delta_{i}, \quad \lambda_{\alpha, i}^{(0)}=V(\alpha+i \omega), \quad Z\left(\Psi_{\alpha, i}^{(0)}\right)=i, \quad-\infty<i<\infty .
$$

Поэтому $\Phi_{\alpha, m}^{(0)}=\Psi_{\alpha, 0}^{(0)}, \Lambda_{\alpha}^{(0)}=V(\alpha)$ и

$$
H_{\alpha} \Psi_{\alpha, i}^{(0)}=V(\alpha+i \omega) \Psi_{\alpha, i}^{(0)}+h_{\alpha, i}^{(0)}
$$

7 УМН, т. 54, вып. 1 
где $h_{\alpha, i}^{(0)}$ - погрешность. На следуюшем шаге хотелось бы положить, в соответствии с обычной теорией возмущений,

$$
\Psi_{\alpha, i}^{(1)}=\Psi_{\alpha, i}^{(0)}+\sum_{j \neq i} \frac{\left(h_{\alpha, i}^{(0)}, \Psi_{\alpha, i}^{(0)}\right)}{\lambda_{\alpha, i}^{(0)}-\lambda_{\alpha, j}^{(0)}} \Psi_{\alpha, j}^{(0)} .
$$

Эти формулы неприменимы, если знаменатель $\lambda_{\alpha, i}^{(0)}-\lambda_{\alpha, j}^{(0)}$ равен нулю или слишком мал. При таких значениях $\alpha$ мы имеем резонанс между $\Psi_{\alpha, i}^{(0)}$ и $\Psi_{\alpha, j}^{(0)}$. Это означает, что на следуюшем шаге приближенные с.ф.. в главном порядке должны быть линейными комбинациями невозмушенных $\Psi_{\alpha, i}^{(0)}$ и $\Psi_{\alpha, j}^{(0)}$. На первом шаге нашей индуктивной процедуры резонанс происходит около тех $\alpha$, где $V(\alpha)=V(\alpha+\omega)$. В классе функций $V$, которые мы рассматриваем, имеется две таких точки резонанса $\alpha_{1}^{(0)}, \alpha_{2}^{(0)}$, где убываюшая ветвь $V(\alpha)$ пересекается с возрастающей ветвью $V(\alpha+\omega)$ и наоборот (см. рис. 4).

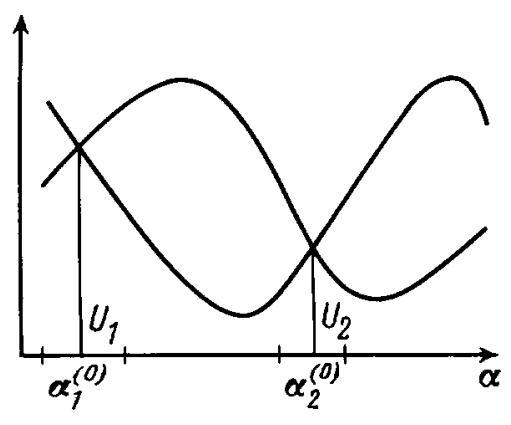

Рис. 4

Поскольку мы должны построить $\Phi_{\alpha}^{(1)}$, мы можем пользоваться формулами $(21)$ вне некоторых окрестностей $U_{1}, U_{2}$ точек $\alpha_{1}^{(0)}, \alpha_{2}^{(0)}$. Внутри же этих окрестностей невозмушенные с.з. близки между собой. Для таких $\alpha$ мы будем строить новые приближенные с.ф., которые в главном порядке представляют собой линейные комбинации невозмущенных с.ф. с вполне определенными весами. Если $\Psi_{\alpha, i}^{(0)}$ и $\Psi_{\alpha, i}^{(0)}=S \Psi_{\alpha+\omega, j}^{(0)}$ имеют близкие приближенные с.з., то мы полагаем

$$
\begin{aligned}
& \Psi_{\alpha, i}^{(1)}=a_{11}\left(\Psi_{\alpha, i}^{(0)}+\sum_{k \neq i, j} \frac{\left(h_{\alpha, i}^{(0)}, \Psi_{\alpha, k}^{(0)}\right)}{\lambda_{\alpha, i}^{(0)}-\lambda_{\alpha, k}^{(0)}} \Psi_{\alpha, k}^{(0)}\right)+a_{12}\left(\Psi_{\alpha, j}^{(0)}+\sum_{k \neq i, j} \frac{\left(h_{\alpha, j}^{(0)}, \Psi_{\alpha, k}^{(0)}\right)}{\lambda_{\alpha, j}^{(0)}-\lambda_{\alpha, k}^{(0)}} \Psi_{\alpha, k}^{(0)}\right), \\
& \Psi_{\alpha, j}^{(1)}=a_{21}\left(\Psi_{\alpha, i}^{(0)}+\sum_{k \neq i, j} \frac{\left(h_{\alpha, i}^{(0)}, \Psi_{\alpha, k}^{(0)}\right)}{\lambda_{\alpha, i}^{(0)}-\lambda_{\alpha, k}^{(0)}} \Psi_{\alpha, k}^{(0)}\right)+a_{22}\left(\Psi_{\alpha, j}^{(0)}+\sum_{k \neq i, j} \frac{\left(h_{\alpha, j}^{(0)}, \Psi_{\alpha, k}^{(0)}\right)}{\lambda_{\alpha, j}^{(0)}-\lambda_{\alpha, k}^{(0)}} \Psi_{\alpha, k}^{(0)}\right) .
\end{aligned}
$$

Матрища $\left\|a_{i, j}\right\|$ ортогональна и приводит матрищу

$$
\left\|\begin{array}{cc}
\lambda_{\alpha, i}^{(0)} & \left(\Psi_{\alpha, i}^{(0)}, \Psi_{\alpha, j}^{(0)}\right) \\
\left(\Psi_{\alpha, i}^{(0)}, \Psi_{\alpha, j}^{(0)}\right) & \lambda_{\alpha, j}^{(0)}
\end{array}\right\|
$$




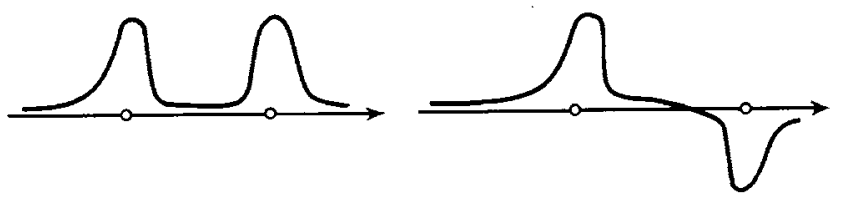

Рис. 5

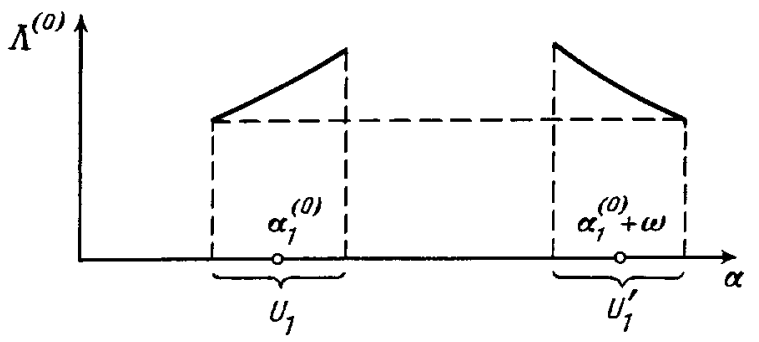

Рис. 6

к диагональному виду. Существенньй носитель новых приближенных с.ф. полагается равным $Z\left(\Psi_{\alpha, i}^{(1)}\right)=Z\left(\Psi_{\alpha, j}^{(1)}\right)=0 \cup 1$, их вид представлен схематически на рис. 5 .

Обсудим подробнее разницу между $\Lambda_{\alpha}^{(0)}$ и $\Lambda_{\alpha}^{(1)}$. Рассмотрим для определенности окрестность $U_{1}$. До того, как строить новые приближенные с.ф., мы имели две окрестности $U_{1}, U_{1}^{\prime}$ и графики $\Lambda_{\alpha}^{(0)}=V(\alpha)$ на них. Выберем эти окрестности $U_{1}, U_{1}^{\prime}$ так, чтобы графики $\Lambda_{\alpha}^{(0)}$ проектировались на один и тот же интервал на оси $\Lambda^{(0)}$ (см. рис. 6$)$. На следуюшем шаге индукции мы имеем две новые приближенные с.ф., вид которых представлен на рис. 5 , с различаюшимися с.з. Функция $\Lambda_{\alpha}^{(0)}$ становится двузначной на $U_{1}$ (см. рис. 7 ) и не принимает вообще значений на $U_{1}^{\prime}$. Это связано с тем, что для $\alpha^{\prime} \in U_{1}^{\prime}$ не существует приближенных с.ф., для которых $\operatorname{pos}\left(\Psi_{\alpha^{\prime}, i}^{(1)}\right)=0$. Если $0 \in Z\left(\Psi_{\alpha^{\prime}, i}^{(1)}\right)$, то другая точка с.н. лежит слева от нуля.

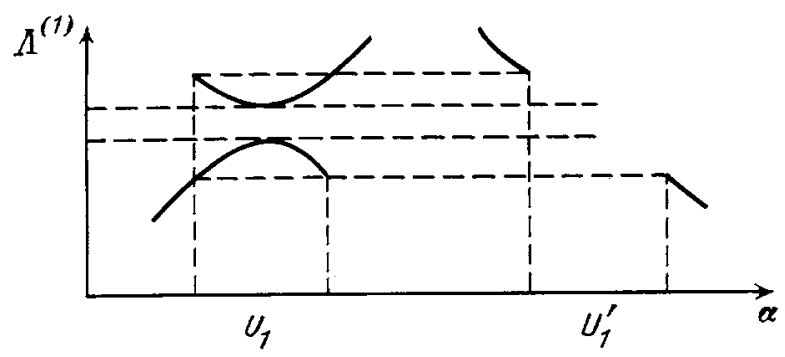

Рис. 7

Вид $\Lambda_{\alpha}^{(1)}$ также важен. На $U_{1}$ ее графики состоит из двух ветвей. Ветви, примыкающие к концам $U_{1}^{\prime}$, принимают в этих концах те же значения, что и ветви $\Lambda_{\alpha}^{(1)}$, опреде- 


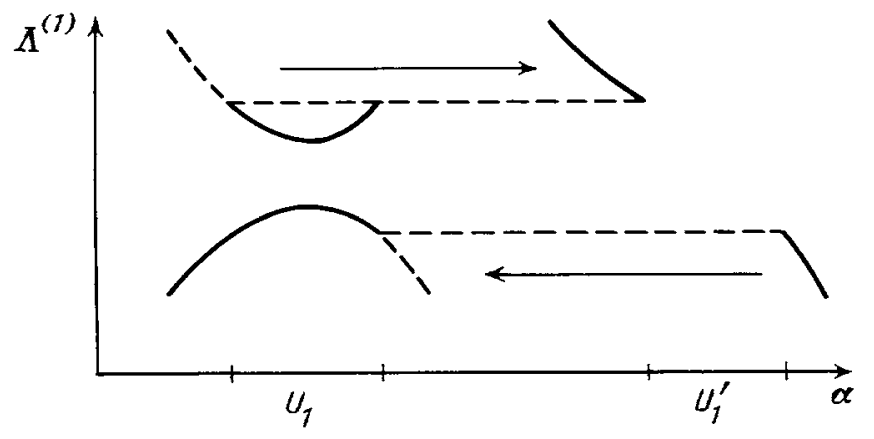

Рис. 8

ленные на $U_{1}$. Более того, если взять график $\Lambda_{\alpha}^{(1)}$ около границы $U_{1}^{\prime}$ и сдвинуть его на $-\omega$, то он будет представлять собой $C^{2}$-гладкое продолжение соответствующей ветви на $U_{1}$ (см. рис. 8). Ясно также, что $\Lambda_{\alpha}^{(1)}$ и $\Lambda_{\alpha+\omega}^{(1)}$ не пересекаются нигде, кроме конщов ветвей $\Lambda_{\alpha}^{(1)}$. Уже на этом шаге теории возмушений полезно проследить появление на оси $\Lambda$ интервалов, где нет значений $\Lambda_{\alpha}^{(1)}$, так назьваемых запрешенных зон. Они возникают вследствие резонансов, и мы увидим далее, что вся конструкция приводит к спектру, сосредоточенному на Канторовом множестве положительной меры с бесконечным числом запрешенных зон.
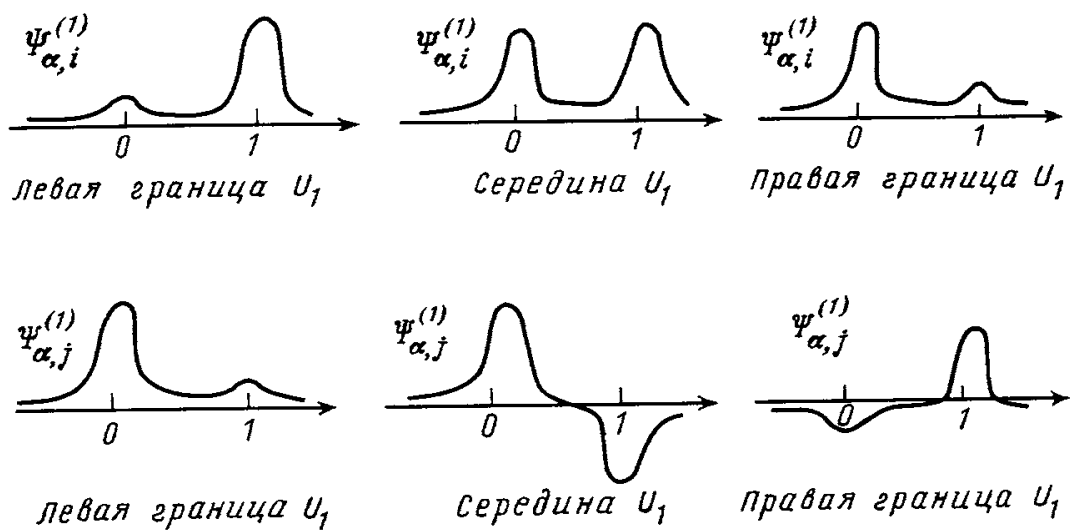

Рис. 9

Обсудим теперь выбор размеров окрестностей $U_{1}, U_{1}^{\prime}$. Легко посчитать, что форма $\Psi_{\alpha, i}^{(1)}$ и $\Psi_{\alpha, j}^{(1)}$ по мере изменения $\alpha$ вдоль $U_{1}$ меняется следуюшим образом (рис. 9 ). В концах $U_{1}$ приближенные с.ф. $\Psi_{\alpha, i}^{(1)}, \Psi_{\alpha, j}^{(1)}$ имеют только один существенньй пик, и эта форма продолжается в нерезонансную область. Поэтому выбор границ $U_{1}$ неоднозначен и может меняться в определенных пределах. Иногда полезно гранищы $U_{1}$ немного сдвигать, если пересечения графиков соответствующих функций происходят слишком близко к их концам (см. далее). 
Теперь мы сформулируем индуктивные предположения на $n$-м шаге нашей индуктивной процедуры. Допустим, что мы имеем функцию $\Phi_{\alpha}^{(n)}$, значения которой представляют собой конечные множества (возможно, пустые) приближенных нормированных с.ф. $\Psi_{\alpha, i}^{(n)}$, имеюших с.н. $Z\left(\Psi_{\alpha, i}^{(n)}\right)$, причем $\operatorname{pos}\left(Z\left(\Psi_{\alpha, i}^{(n)}\right)\right)=0$. Кроме того, каждая приближенная с.ф. $\Psi_{\alpha, i}^{(n)}$ имеет полньй конечный носитель, вне которого она тождественно равна нулю. Мы обозначим его $F Z\left(\Psi_{\alpha, i}^{(n)}\right)$. Полный носитель растет с ростом $n$, в то время как с.н. $Z\left(\Psi_{\alpha, i}^{(n)}\right)$ стабилизируется. $F Z\left(\Psi_{\alpha, i}^{(n)}\right)$ представляет собой конечный отрезок.

Строим базис в $l^{2}$ по формуле $\left\{\Psi_{\alpha, i}^{(n)}\right\}=\bigcup_{-\infty<m<\infty} S^{m} \Phi_{\alpha+m \omega}^{(n)}$. Этот базис неортогональньй и становится ортогональным только в пределе $n \rightarrow \infty$. Утверждение о том, что $\left(\Psi_{\alpha, i}^{(n)}\right)$ образует базис, можно считать еще одним индуктивным предположением. Для каждой приближенной с.ф. $\Psi_{\alpha, i}^{(n)} \in \Phi_{\alpha}^{(n)}$ задано приближенное с.з. $\lambda_{\alpha, i}^{(n)}$ так, что

$$
H_{\alpha} \Psi_{\alpha, i}^{(n)}=\lambda_{\alpha, i}^{(n)} \Psi_{\alpha, i}^{(n)}+b_{\alpha, i}^{(n)}+h_{\alpha, i}^{(n)},
$$

где $b_{\alpha, i}^{(n)}$ и $h_{\alpha, i}^{(n)}$ - погрешности. Расшепление всей погрешшости на две части сушественно. Пусть $F Z\left(\Psi_{\alpha, i}^{(n)}\right)$ есть отрезок $\left[m_{1}, m_{2}\right]$. Тогда $b_{\alpha, i}^{(n)}$ отлична от нуля только в точках $m_{1}-1, m_{1}$ и $m_{2}, m_{2}+1$, а $h_{\alpha, i}^{(n)}$ равна нулю в этих точках. Этим $b_{\alpha, i}^{(n)}$ и $h_{\alpha, i}^{(n)}$ определены однозначно и $h_{\alpha, i}^{(n)}(m)$ равно нулю при всех $m$ вне отрезка $\left[m_{1}+2, m_{2}-2\right]$.

Полагаем $\Lambda_{\alpha}^{(n)}=\left\{\lambda_{\alpha, i}^{(n)} \mid \Psi_{\alpha, i}^{(n)} \in \Phi_{\alpha}^{(n)}\right\}$ и $\bigcup_{-\infty<m<\infty} \Lambda_{\alpha+m \omega}^{(n)}$ есть приближенньй спектр $H_{\alpha}$.

Как уже отмечалось вьше, из того, что $V$ принимает каждое значение не более чем в двух точках, следует, что с.н. $Z\left(\Psi_{\alpha, i}^{(n)}\right)$ имеет специальную иерархическую структуру, которая связана со структурой резонансов. Мы опишем ее снова, и это можно считать еще одним индуктивным предположением. А именно,

$$
Z\left(\Psi_{\alpha, i}^{(n)}\right)=Z_{1} \cup\left(Z_{1}+m_{r}\right), \quad Z_{1}=Z_{2} \cup\left(Z_{2} \cup m_{r-1}\right)
$$

и т. д. Положительные числа $m_{1}, m_{2}, \ldots$ назьваются ширинами резонансов. Из конструкции будет следовать, что $m_{i}$ растут очень быстро, $m_{i}$ принимает значения порядка $\exp \left\{\right.$ const $\left.\cdot m_{i-1}\right\}$. Такая иерархическая структура означает, в частности, что

$$
\#\left\{Z\left(\Psi_{\alpha, i}^{(n)}\right)\right\}=2^{r},
$$

где $r$ - число резонансов, в которых участвует данная приближенная с.ф. (см. далее).

Теперь мы опишем предположения, касаюшиеся формы $\Lambda_{\alpha}^{(n)}$, которая не слишком сильно отличается от формы $\Lambda_{\alpha}^{(n)}$. Допустим, что на оси $\Lambda$ имеется несколько отрезков, обозначаемых далее $\Delta_{k, s}^{(n)}$, индекс $s$ принимает значения $1,2,3$. Для каждого $\Delta_{k, 2}^{(n)}$ отрезок $\Delta_{k, 1}^{(n)}$ примыкает к $\Delta_{k, 2}^{(n)}$ слева, а отрезок $\Delta_{k, 3}^{(n)}-$ справа. Отрезок $\Delta_{k, 2}^{(n)}$ гораздо длиннее примыкающих к нему отрезков: $l\left(\Delta_{k, 1}^{(n)}\right), l\left(\Delta_{k, 3}^{(n)}\right) \leqslant \operatorname{const} \varepsilon n^{-3} l\left(\Delta_{k, 2}^{(n)}\right)$. Выбop const будет описан позже. В действительности, левые части этого неравенства гораздо меньше. 
Для каждого $\Delta_{k, 2}^{(n)}$ можно найти два отрезка $U_{k, 2,1}^{(n)}, U_{k, 2,2}^{(n)} \subset S^{1}$ так, что среди значений $\Lambda_{\alpha}^{(n)}$ имеются $C^{2}$-функция $\Lambda_{\alpha}^{(n)}(k, 2,1)$, заданная на $U_{k, 2,1}^{(n)}$, и $C^{2}$-функция $\Lambda_{\alpha}^{(n)}(k, 2,2)$, заданная на $U_{k, 2,2}^{(n)}$, и при этом

$\left.\mathrm{i}_{0}\right)$ с.н. всех приближенных с.ф. $\Psi_{\alpha, i}^{(n)}$, отвечающих этим ветвям, одни и те же, т.е. не зависят от $\alpha$;

$\left.\mathrm{i}_{1}\right) \Lambda_{\alpha}^{(n)}(k, 2,1)$ монотонно возрастает, $\Lambda_{\alpha}^{(n)}(k, 2,2)$ монотонно убьвает;

$\left.\mathrm{i}_{2}\right)$ функция $\Lambda_{\alpha}^{(n)}(k, 2,1)\left(\Lambda_{\alpha}^{(n)}(k, 2,2)\right)$ отображает гомеоморфно $U_{k, 2,1}^{(n)}\left(U_{k, 2,2}^{(n)}\right)$ на $\Delta_{k, 2}^{(n)} ;$ прообраз $\Delta_{k, 2}^{(n)}$ при отображении $\left(\Lambda^{(n)}\right)^{-1}$ есть $U_{k, 2,1}^{(n)} \cup U_{k, 2,2}^{(n)}$;

i $) \frac{d}{d \alpha} \Lambda_{\alpha}^{(n)}(k, 2,1) \geqslant\left(b_{1} \varepsilon\right)^{n}, \frac{d}{d \alpha} \Lambda_{\alpha}^{(n)}(k, 2,2) \leqslant-\left(b_{1} \varepsilon\right)^{n}$;

$\left.\mathrm{i}_{4}\right)\left|\frac{d^{2} \Lambda_{\alpha}^{(n)}(k, 2,1)}{d \alpha^{2}}\right| \leqslant\left(B_{2} \varepsilon\right)^{-n},\left|\frac{d^{2} \Lambda_{\alpha}^{(n)}(k, 2,2)}{d \alpha^{2}}\right| \leqslant\left(B_{2} \varepsilon\right)^{-n}$.

Здесь $b_{1}, B_{2}$ - постоянные, не зависящие от $n$, о которых будет сказано позже. Типичный вид $\Lambda_{\alpha}^{(n)}(k, 2,1), \Lambda_{\alpha}^{(n)}(k, 2,2)$ представлен на рис. 10.

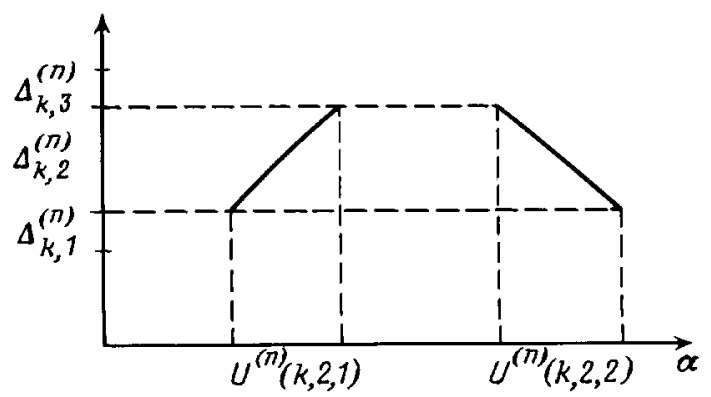

Рис. 10

Рассмотрим теперь $\Delta_{k, 3}^{(n)}$ и $\Delta_{k+1,1}^{(n)}$. Интервал между ними представляет собой приближенную запрешенную зону на $n$-м шаге. Можно найти интервал $U_{k, 3}^{(n)} \subset S^{1}$ так, что среди значений $\Lambda_{\alpha}^{(n)}$ имеется $C^{2}$-функция $\Lambda_{\alpha}^{(n)}(k, 3)$, определенная на $U_{k, 3}^{(n)}$ и такая, что

j1 $\Lambda_{k, 3}^{(n)}$ отображает $U_{k, 3}^{(n)}$ на $\Delta_{k, 3}^{(n)}$ и при этом принимает одинаковые значения в конщах $U_{k, 3}^{(n)} ;$ прообраз $\Delta_{k, 3}^{(n)}$ при отображении $\left(\Lambda^{(n)}\right)^{-1}$ есть $U_{k, 3}^{(n)}$;

$\left.\mathrm{j}_{2}\right)$ с.н. различных приближенных $\Psi_{\alpha, i}^{(n)}, \alpha \in U_{k, 3}^{(n)}$, один и тот же и не зависит от $\alpha$;

јз) функция $\Lambda_{k, 3}^{(n)}$ вогнута и

$$
\frac{d^{2} \Lambda_{k, 3}^{(n)}}{d \alpha^{2}} \leqslant-\left(B_{3} \varepsilon\right)^{n}
$$


Аналогичным образом, для отрезка $\Delta_{k+1,1}^{(n)}$ найдется окрестность $U_{k+1,1}^{(n)} \subset S^{1}$ и ветвь $\Lambda_{k+1,1}^{(n)}$ функции $\Lambda_{\alpha}^{(n)}$, заданная на $U_{k+1,1}^{(n)}$ и такая, что

$\left.\mathrm{j}_{1}^{\prime}\right) \Lambda_{(k+1,1)}^{(n)}$ отображает $U_{k+1,1}^{(n)}$ на $\Delta_{k+1,1}^{(n)}$ и принимает одинаковые значения в концах $U_{k+1,1}^{(n)}$; прообраз $U_{k+1,1}^{(n)}$ при отображении $\left(\Lambda^{(n)}\right)^{-1}$ есть $U_{k+1,1}^{(n)}$;

$\left.\mathrm{j}_{2}^{\prime}\right)$ с.н. различных с.ф. $\Psi_{\alpha, i}^{(n)}, \alpha \in U_{k+1,1}^{(n)}$, один и тот же и не зависит от $\alpha$;

$\left.\mathrm{j}_{3}^{\prime}\right)$ функция $\Lambda_{k+1,1}^{(n)}$ выпукла и

$$
\frac{d^{2} \Lambda^{(n)}(k+1,1)}{d \alpha^{2}} \geqslant\left(B_{3} \varepsilon\right)^{n},
$$

$B_{3}$ - еще одна постоянная той же природы, что и $b_{1}, B_{2}$.

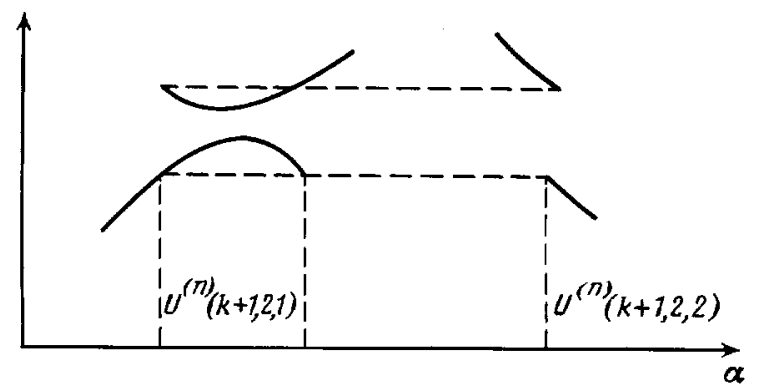

Рис. 11

Типичньй вид $\Lambda_{\alpha}^{(n)}(k, 3)$ и $\Lambda_{\alpha}^{(n)}(k+1,1)$ представлен на рис. 11. Последний справа сегмент $\Delta_{k_{1}, 3}^{(n)}$, правая граница которого обозначается $\Lambda_{\max }^{(n)}$, для нас особенно важен ввиду содержания $\S 1$ и $\S 2$. К этому интервалу не примыкает следующий интервал $\Delta_{k_{1}+1,1}^{(n)}$ и имеется окрестность $U_{\alpha}^{(n)}\left(k_{1}, 3\right)$ и только одна вьпуклая функция $\Lambda_{\alpha}^{(n)}\left(k_{1}, 3\right)$, удовлетворяющая тем же неравенствам $\left.j_{2}\right)$. Аналогичное предположение вьполняется около точки, где $\Lambda_{\alpha}^{(n)}$ принимает минимальное значение. Вся функция $\Lambda_{\alpha}^{(n)}$ есть объединение ветвей $\Lambda_{\alpha}^{(n)}(k, 1), \Lambda_{\alpha}^{(n)}(k, 2), \Lambda_{\alpha}^{(n)}(k, 3)$. Наши предположения описьвают вид $\Lambda_{\alpha}^{(n)}$ на прообразе каждого сегмента $\Delta_{k, s}^{(n)}$. Сушественно также, что ветви $\Lambda_{\alpha}^{(n)}$ не пересекаются с ветвями сдвигов $\Lambda_{\alpha+m \omega}^{(n)}, 0 \leqslant m \leqslant n$, кроме, быть может, конщов. Более точно, $\Lambda_{\alpha+m \omega}^{(n)}(k+1,2,2)$ является $C^{2}$ - продолжением $\Lambda_{\alpha+m \omega}^{(n)}(k+1,1)$ и $\Lambda_{\alpha+m \omega}^{(n)}(k+1,2,1)$ является $C^{2}$-продолжением $\Lambda_{\alpha+m \omega}^{(n)}(k, 3)$ (см. рис. 12). Отсутствие пересечений означает, что к $n$-му шагу мы уже учли все резонансы ширины, не превосходяшей $n$.

Основная индуктивная процедура состоит в построении новых приближенных с.ф. $\Psi_{\alpha, i}^{(n+1)}$, функции $\Phi_{\alpha}^{(n+1)}$, соответствуюших приближенных с.з. $\lambda_{\alpha, i}^{(n+1)}$ и функции $\Lambda_{\alpha}^{(n+1)}$.

Рассмотрим пересечения графиков $\Lambda_{\alpha}^{(n)}$ и $\Lambda_{\alpha+n \omega}^{(n)}$, что, очевидно, сводится к пересечению их ветвей, описанных выше. Ясно, что эти пересечения могут изучаться 


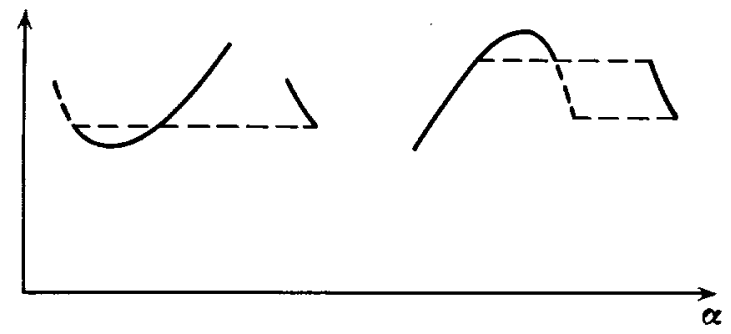

Рис. 12

отдельно для каждого из отрезков $\Delta_{k, s}^{(n)}$. Поскольку в выборе их концов есть определенньй произвол, мы всегда можем считать, что эти пересечения происходят во внутренних точках всех отрезков. Ясно также, что благодаря иррациональности $\omega$ пересечения возможны

либо между ветвями $\Lambda_{\alpha}^{(n)}(k, 2,1)$ и $\Lambda_{\alpha+n \omega}^{(n)}(k, 2,2)$ или $\Lambda_{\alpha}^{(n)}(k, 2,2)$ и $\Lambda_{\alpha+n \omega}^{(n)}(k, 2,1)$ (см. рис. $13(\mathrm{a}))$,

либо между $\Lambda_{\alpha}^{(n)}(k, 3)$ и $\Lambda_{\alpha+m \omega}^{(n)}(k, 3)$ (см. рис. $\left.13(\mathrm{~b})\right)$.

либо между $\Lambda_{\alpha}^{(n)}(k, 1)$ и $\Lambda_{\alpha+m \omega}^{(n)}(k, 1)($ см. рис. $13(\mathrm{c}))$.
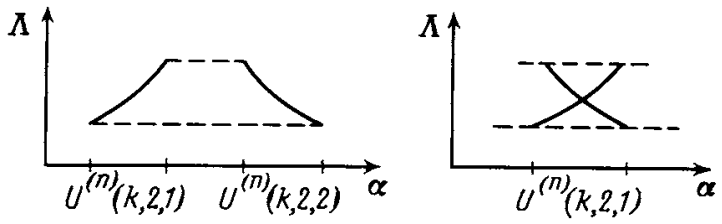

(a)

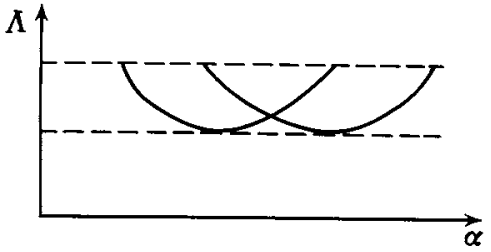

(b)

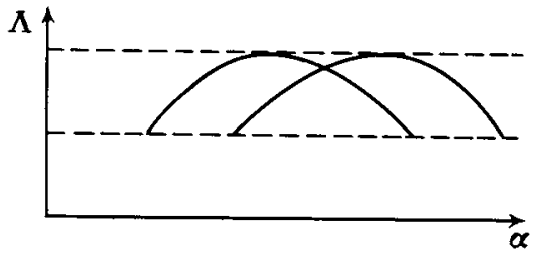

(c)

Рис. 13

Пусть $C_{4}=C_{4}(\omega, \alpha, \varepsilon)$ - еше одна достаточно большая постоянная, выбор которой также обсуждается позже. Окрестность радиуса $\left(C_{4} \varepsilon\right)^{n}$ точки пересечения $\Lambda_{\alpha}^{(n)} \cap \Lambda_{\alpha+n \omega}^{(n)}$ будем назьвать резонансной зоной. Возьмем $\Psi_{\alpha, i}^{(n)} \in \Phi_{\alpha}^{(n)}$ и соответствующее $\lambda_{\alpha, i}^{(n)}$. Назовем $\Psi_{\alpha, i}^{(n)}$ нерезонансной приближенной с.ф. $($ на $(n+1)$-м шаге нашей процедуры), если для любой точки пересечения $(\lambda, \alpha) \in \Lambda_{\alpha}^{(n)} \cap \Lambda_{\alpha+n \omega}^{(n)}$ справедливо неравенство $\left|\lambda-\lambda_{\alpha, i}^{(n)}\right| \geqslant\left(C_{4} \varepsilon\right)^{n}$. 
В нерезонансном случае мы строим следующее приближение к с.ф. с помошью обычных формул теории возмушений:

$$
\begin{aligned}
\widetilde{\Psi}_{\alpha, i}^{(n+1)} & =\Psi_{\alpha, i}^{(n)}+\sum_{j \neq i} \frac{\left(b_{\alpha, i}^{(n)}+h_{\alpha, i}^{(n)}, \Psi_{\alpha, i}^{(n)}\right)}{\lambda_{\alpha, i}^{(n)}-\lambda_{\alpha, j}^{(n)}} \Psi_{\alpha, j}^{(n)}, \\
\lambda_{\alpha, i}^{(n+1)} & =\lambda_{\alpha, i}^{(n)}+\left(b_{\alpha, i}^{(n)}+h_{\alpha, i}^{(n)}, \Psi_{\alpha, i}^{(n)}\right) .
\end{aligned}
$$

Покажем, что формулы (23), (24) действительно задают следующее приближение к будущей с.ф. и будущему с.з. Мы имеем

$$
H_{\alpha} \widetilde{\Psi}_{\alpha, i}^{(n+1)}=\lambda_{\alpha}^{(n)} \Psi_{\alpha, i}^{(n)}+b_{\alpha, i}^{(n)}+h_{\alpha, i}^{(n)}+\sum_{j \neq i} \frac{\left(b_{\alpha, i}^{(n)}+h_{\alpha, i}^{(n)}, \Psi_{\alpha, i}^{(n)}\right) \lambda_{\alpha, j}^{(n)} \Psi_{\alpha, j}^{(n)}}{\lambda_{\alpha, i}^{(n)}-\lambda_{\alpha, j}^{(n)}}+\widetilde{e}_{\alpha, i}^{(n)}
$$

где $\widetilde{e}_{\alpha, i}^{(n)}$ - погрешность более высокого порядка малости, получаюшаяся от того, что в последнюю сумму мы подставляем соответствующие погрешности $b_{\alpha, i}^{(n)}$ и $h_{\alpha, i}^{(n)}$. Далее, при $\delta \lambda_{\alpha, i}^{(n)}=\left(b_{\alpha, i}^{(n)}+h_{\alpha, i}^{(n)}, \Psi_{\alpha, i}^{(n)}\right)$

$$
\begin{aligned}
H_{\alpha} \widetilde{\Psi}_{\alpha, i}^{(n)}-\lambda_{\alpha, i}^{(n+1)} \widetilde{\Psi}_{\alpha, i}^{(n)} & =H_{\alpha} \widetilde{\Psi}_{\alpha, i}^{(n)}-\lambda_{\alpha, i}^{(n)} \widetilde{\Psi}_{\alpha, i}^{(n)}-\delta \lambda_{\alpha, i}^{(n)} \widetilde{\Psi}_{\alpha, i}^{(n)} \\
& =b_{\alpha, i}^{(n)}+h_{\alpha, i}^{(n)}-\sum_{j}\left(b_{\alpha, i}^{(n)}+h_{\alpha, i}^{(n)}, \Psi_{\alpha, j}^{(n)}\right) \Psi_{\alpha, j}^{(n)}+\widetilde{e}_{\alpha, i}^{(n)}
\end{aligned}
$$

Если бы набор $\left\{\Psi_{\alpha, i}^{(n)}\right\}$ был ортонормированным базисом, то от последнего выражения осталось бы только $\widetilde{e}_{\alpha, i}^{(n)}$. Однако в нашем случае это не так. Введем матрицу скалярных произведений $\delta_{\alpha, i, j}^{(n)}=\left(\Psi_{\alpha, i}^{(n)}, \Psi_{\alpha, j}^{(n)}\right)=\delta_{i, j}+\xi_{\alpha, i, j}^{(n)}$. Это скалярное произведение заведомо равно нулю, если полные носители $F Z\left(\Psi_{\alpha, i}^{(n)}\right), F Z\left(\Psi_{\alpha, j}^{(n)}\right)$ попарно не пересекаются. В противном случае оно мало, и мы вводим еще одно требование, которое также проверяем по индукции: $\left|\xi_{\alpha, i, j}^{(n)}\right| \leqslant\left(C_{5}^{n}\right)^{n}$, где $C_{5}<1$-еше одна постоянная, не зависящая от $n, \varepsilon, \alpha$, т.е. $C_{5}=C_{5}(\omega)$.

Мы полагаем в нерезонансном случае $Z\left(\widetilde{\Psi}_{\alpha, i}^{(n+1)}\right)=Z\left(\Psi_{\alpha, i}^{(n)}\right)$. Полньй носитель $F Z\left(\Psi_{\alpha, i}^{(n+1)}\right)$ будущей приближенной с.ф. $\Psi_{\alpha, i}^{(n+1)}$ получается добавлением одной точки слева и одной точки справа к $F Z\left(\Psi_{\alpha, i}^{(n)}\right)$. После того как $F Z\left(\Psi_{\alpha, i}^{(n+1)}\right)$ выбран, мы рассматриваем ограничение $\widetilde{\Psi}_{\alpha, i}^{(n+1)}$ на $F Z\left(\Psi_{\alpha, i}^{(n+1)}\right)$ и нормируем его так, чтобы норма этого ограничения была равна 1 . Полученную функцию и обозначим $\Psi_{\alpha, i}^{(n+1)}$. Ясно, что $\Psi_{\alpha, i}^{(n+1)}$ равна нулю вне $F Z\left(\Psi_{\alpha, i}^{(n+1)}\right)$. Выражения для $b_{\alpha, i}^{(n+1)}, h_{\alpha, i}^{(n+1)}$ следуют из их определения.

Перейдем к резонансному случаю, когда для $\Psi_{\alpha, i}^{(n)} \in \Phi_{\alpha}^{(n)}$ найдется приближенная с.ф. $\Psi_{\alpha+n \omega, j}^{(n)} \in \Phi_{\alpha}^{(n)}$, для которой $\left|\lambda_{\alpha, i}^{(n)}-\lambda_{\alpha+n \omega, j}^{(n)}\right| \leqslant\left(C_{4} \varepsilon\right)^{n}$ и правьй конец $F Z\left(\Psi_{\alpha, i}^{(n)}\right)$ является соседним с левым конщом $F Z\left(\Psi_{\alpha, i}^{(n)}\right)+n$. В силу наших предположений о $\Lambda_{\alpha}^{(n)}$ это означает, что $\alpha$ лежит в $\left(C_{4} \varepsilon\right)^{n}$-окрестности одной из точек пересечения $\Lambda_{\alpha}^{(n)}$ и $\Lambda_{\alpha+n \omega}^{(n)}$. Такую ситуацию естественно назвать стыковкой приближенных с.ф. Нетрудно показать, что такая $\Psi_{\alpha+n \omega, j}^{(n)}$ только одна, т.е. резонанс возможен 
лишш между двумя приближенными с.ф. В этом сказьвается специфика нашей ситуации, связанная, главным образом, с тем, что мы имеем только одну частоту $\omega$. Мы будем здесь использовать формулы теории возмущений в резонансном случае, которые дают вместо двух приближенных с.ф. $\Psi_{\alpha, i}^{(n)}, \Psi_{\alpha+n \omega, j}^{(n)}$ две новые приближенные с.ф. $\Psi_{\alpha, i}^{(n+1)}, \Psi_{\alpha+n \omega, j}^{(n+1)}$, являюшиеся линейными комбинациями $\Psi_{\alpha, i}^{(n)}$ и $S^{n} \Psi_{\alpha+n \omega, j}^{(n)}$. Аналогичные выражения можно найти в работах [4], [6], [7]. Существенный носитель $\Psi_{\alpha, i}^{(n+1)}$, $\Psi_{\alpha, j}^{(n+1)}$ полагается равным

$$
Z\left(\Psi_{\alpha, i}^{(n)}\right) \cup\left(Z\left(\Psi_{\alpha+n \omega, j}^{(n)}\right)+n\right)=Z\left(\Psi_{\alpha, i}^{(n)}\right) \cup\left(Z\left(\Psi_{\alpha, i}^{(n)}\right)+n\right)
$$

поскольку резонанс возможен только между приближенными с.ф. с одним и тем же с.н. Отсюда вытекает иерархическая структура с.н., о которой упоминалось вьше. Новые приближенные с.ф. строятся с помошью тех же формул, что и выше, т.е.

$$
\begin{aligned}
\widetilde{\Psi}_{\alpha, i}^{(n+1)}= & a_{11}\left(\Psi_{\alpha, i}^{(n)}+\sum_{k \neq i, j} \frac{\left(b_{\alpha, i}^{(n)}+h_{\alpha, i}^{(n)}, \Psi_{\alpha, k}^{(n)}\right)}{\lambda_{\alpha, i}^{(n)}-\lambda_{\alpha, k}^{(n)}} \Psi_{\alpha, k}^{(n)}\right) \\
& +a_{12}\left(\Psi_{\alpha, i}^{(n)}+\sum_{k \neq i, j} \frac{\left(b_{\alpha, j}^{(n)}+h_{\alpha, j}^{(n)}, \Psi_{\alpha, k}^{(n)}\right)}{\lambda_{\alpha, j}^{(n)}-\lambda_{\alpha, k}^{(n)}} \Psi_{\alpha, k}^{(n)}\right) \\
\widetilde{\Psi}_{\alpha, j}^{(n+1)}= & a_{21}\left(\Psi_{\alpha, i}^{(n)}+\sum_{k \neq i, j} \frac{\left(b_{\alpha, i}^{(n)}+h_{\alpha, i}^{(n)}, \Psi_{\alpha, k}^{(n)}\right)}{\lambda_{\alpha, i}^{(n)}-\lambda_{\alpha, k}^{(n)}} \Psi_{\alpha, k}^{(n)}\right) \\
& +a_{22}\left(\Psi_{\alpha, i}^{(n)}+\sum_{k \neq i, j} \frac{\left(b_{\alpha, j}^{(n)}+h_{\alpha, j}^{(n)}, \Psi_{\alpha, k}^{(n)}\right)}{\lambda_{\alpha, j}^{(n)}-\lambda_{\alpha, k}^{(n)}} \Psi_{\alpha, k}^{(n)}\right)
\end{aligned}
$$

Уравнение для новых приближенных с.з. $\mu$ есть квадратное уравнение

$$
\begin{aligned}
& \left(\lambda_{\alpha, i}^{(n)}-\mu\right)\left(\lambda_{\alpha, j}^{(n)}-\mu\right)+\left(b_{\alpha, i}^{(n)}+h_{\alpha, i}^{(n)}, \Psi_{\alpha, i}^{(n)}\right) \cdot\left(\lambda_{\alpha, j}^{(n)}-\mu\right)+\left(\lambda_{\alpha, i}^{(n)}-\mu\right)\left(b_{\alpha, j}^{(n)}+h_{\alpha, j}^{(n)}, \Psi_{\alpha, j}^{(n)}\right) \\
& \quad+\left(b_{\alpha, i}^{(n)}+h_{\alpha, i}^{(n)}, \Psi_{\alpha, i}^{(n)}\right)\left(b_{\alpha, j}^{(n)}+h_{\alpha, j}^{(n)}, \Psi_{\alpha, j}^{(n)}\right)-\left(b_{\alpha, i}^{(n)}+h_{\alpha, i}^{(n)}, \Psi_{\alpha, j}^{(n)}\right)\left(b_{\alpha, j}^{(n)}+h_{\alpha, j}^{(n)}, \Psi_{\alpha, i}^{(n)}\right)=0 .
\end{aligned}
$$

Его решения имеют вид

$$
\begin{aligned}
\mu= & \frac{\lambda_{\alpha, i}^{(n)}-\lambda_{\alpha, j}^{(n)}}{2}+\frac{\left(b_{\alpha, i}^{(n)}+h_{\alpha, i}^{(n)}, \Psi_{\alpha, i}^{(n)}\right)+\left(b_{\alpha, j}^{(n)}+h_{\alpha, j}^{(n)}, \Psi_{\alpha, j}^{(n)}\right)}{2} \\
\pm & {\left[\left(\frac{\lambda_{\alpha, i}^{(n)}+\lambda_{\alpha, j}^{(n)}}{2}+\frac{\left(b_{\alpha, i}^{(n)}+h_{\alpha, i}^{(n)}, \Psi_{\alpha, i}^{(n)}\right)+\left(b_{\alpha, j}^{(n)}+h_{\alpha, j}^{(n)}, \Psi_{\alpha, j}^{(n)}\right)}{2}\right)^{2}\right.} \\
& -\left(\lambda_{\alpha, i}^{(n)} \lambda_{\alpha, j}^{(n)}+\lambda_{\alpha, i}^{(n)}\left(b_{\alpha, j}^{(n)}+h_{\alpha, j}^{(n)}, \Psi_{\alpha, j}^{(n)}\right)+\lambda_{\alpha, j}^{(n)}\left(b_{\alpha, i}^{(n)}+h_{\alpha, i}^{(n)}, \Psi_{\alpha, i}^{(n)}\right)\right. \\
& +\left(b_{\alpha, i}^{(n)}+h_{\alpha, i}^{(n)}, \Psi_{\alpha, i}^{(n)}\right)\left(b_{\alpha, j}^{(n)}+h_{\alpha, j}^{(n)}, \Psi_{\alpha, j}^{(n)}\right) \\
& \left.\left.-\left(b_{\alpha, i}^{(n)}+h_{\alpha, i}^{(n)}, \Psi_{\alpha, j}^{(n)}\right)\left(b_{\alpha, j}^{(n)}+h_{\alpha, j}^{(n)}, \Psi_{\alpha, i}^{(n)}\right)\right)\right]^{1 / 2} .
\end{aligned}
$$



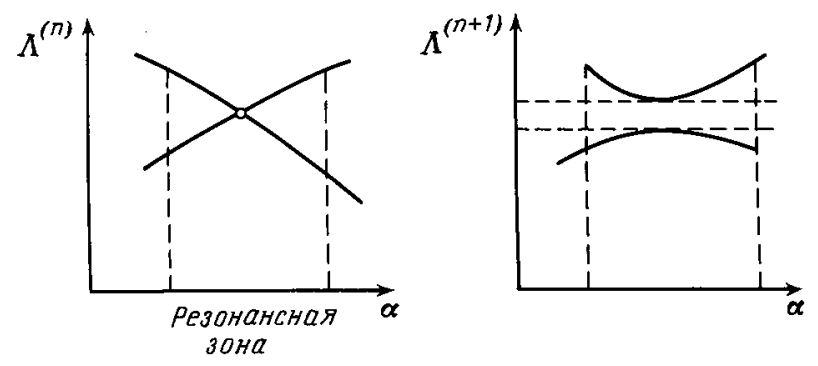

Рис. 14

Разные ветви функции $\Lambda_{\alpha}^{(n+1)}$ соответствуют разньм выборам знака в (26). Рис. 14 показьвает изменение в форме $\Lambda_{\alpha}^{(n)}$ при резонансе и образование новой запрешенной зоны.

Согласно формулам

$$
b_{\alpha, i}^{(n)}\left(m_{0}\right)=-\varepsilon \Psi_{\alpha, i}^{(n)}\left(m_{0}-1\right), \quad b_{\alpha, j}^{(n)}\left(m_{0}\right)=-\varepsilon \Psi_{\alpha, i}^{(n)}\left(m_{0}+1\right)
$$

Кроме того, еще только $b_{\alpha, i}^{(n)}\left(m_{0}-1\right), b_{\alpha, j}^{(n)}\left(m_{0}+1\right)$ могут быть отличны от нуля, а остальные компоненты этих векторов равны нулю. Напомним также, что $\left(b_{\alpha, i}^{(n)}, \Psi_{\alpha, i}^{(n)}\right)$ $=\left(b_{\alpha, j}^{(n)}, \Psi_{\alpha, j}^{(n)}\right)=0$. Из конструкции следует, что $h_{\alpha, i}^{(n)}, h_{\alpha, j}^{(n)}$ имеют более высокий порядок малости, чем $b_{\alpha, i}^{(n)}, b_{\alpha, j}^{(n)}$. Поэтому в главном порядке выражение в квадратных скобках в (26) имеет вид

$$
\begin{aligned}
\left(\frac{\lambda_{\alpha, i}^{(n)}-\lambda_{\alpha, j}^{(n)}}{2}\right)^{2}+\left(b_{\alpha, i}^{(n)},\right. & \left.\Psi_{\alpha, j}^{(n)}\right)\left(b_{\alpha, j}^{(n)}, \Psi_{\alpha, i}^{(n)}\right) \\
& =\left(\frac{\lambda_{\alpha, i}^{(n)}-\lambda_{\alpha, j}^{(n)}}{2}\right)^{2}+\varepsilon^{2}\left(\Psi_{\alpha, i}^{(n)}\left(m_{0}-1\right) \Psi_{\alpha, j}^{(n)}\left(m_{0}\right)\right)^{2}
\end{aligned}
$$

Произведение $\Psi_{\alpha, i}^{(n)}\left(m_{0}-1\right) \Psi_{\alpha, j}^{(n)}\left(m_{0}\right)$ есть аналог интеграла перекрытия в теории резонансов. Оно может быть оценено на основании асимптотики убывания приближенных с.ф. Этот вопрос будет обсуждаться более подробно в следуюшем параграфе. Здесь же мы только отметим, что возможны нетипичные точки $m_{0}$, где $\Psi_{\alpha, i}^{(n)}\left(m_{0}-1\right)$ или $\Psi_{\alpha, j}^{(n)}\left(m_{0}\right)$ аномально малы. Эти точки встречаются достаточно редко, и в таких случаях мы должны слегка видоизменить нашу процедуру, организовав процесс таким образом, чтобы стыковка всегда происходила в типичных точках.

Мы определяем теперь $\Psi_{\alpha, i}^{(n+1)}, \Psi_{\alpha, j}^{(n)}$ как ограничения $\widetilde{\Psi}_{\alpha, i}^{(n+1)}, \widetilde{\Psi}_{\alpha, j}^{(n)}$ на множество, получаемое из $F Z\left(\Psi_{\alpha, i}^{(n+1)}\right) \cup F Z\left(\Psi_{\alpha, j}^{(n)}\right)$ добавлением крайних точек, и нормировку. Во всех случаях расстояние от $Z\left(\Psi_{\alpha, i}^{(n+1)}\right)$ до границы $F Z\left(\Psi_{\alpha, i}^{(n+1)}\right)$ равно $n+1$.

Новая функция $\Lambda_{\alpha}^{(n+1)}$ отличается по форме от $\Lambda_{\alpha}^{(n)}$ в окрестностях точек пересечения $\Lambda_{\alpha}^{(n)}$ и $\Lambda_{\alpha+n \omega}^{(n)}$ (см. рис. 11$)$, а вне этих окрестностей представляет собой малое возмущение $\Lambda_{\alpha}^{(n)}$. 
Тем самым индуктивная процедура полностью определена. Прямые оценки показывают, что все погрешности стремятся к нулю, и в пределе мы получаем точные с.ф. оператора $H_{\alpha}$.

\section{$\S$ 4. Экспоненциальное убывание приближенных собственных функций и другие асимптотические соотношения}

Пользуясь формулами индуктивной процедуры, рассмотрим снова вопрос об экспоненциальном убывании приближенных с.ф. Именно это свойство делает всю индуктивную процедуру сходящейся.

Мы изучаем отношение $\frac{\Psi_{\alpha, i}^{(n+1)}(m+1)}{\Psi_{\alpha, i}^{(n+1)}(m)}$ для точек $m$, принадлежаших полному носителю $F Z\left(\Psi_{\alpha, i}^{(n)}\right)$, но отстоящих от края $F Z\left(\Psi_{\alpha, i}^{(n+1)}\right)$ на расстояние, превосходящее 2 . Достаточно рассматривать только нерезонансную ситуацию. При таких $m \Psi_{\alpha, i}^{(n+1)}(m)$ совпадает с точностью до множителя с $\widetilde{\Psi}_{\alpha, i}^{(n+1)}(m)$. Поэтому вопрос имеет смысл и для функции $\widetilde{\Psi}_{\alpha, i}^{(n+1)}(m)$. В наших дальнейших рассуждениях встречаются величины, допускающие оценки сверху типа $(G \cdot \varepsilon)^{n}$, где $G$ - величина порядка 1 . Такой порядок малости имеют, например, основные погрешности $b_{\alpha, i}^{(n)}$, т.е. значения приближенных с.ф. около конщов $F Z\left(\Psi_{\alpha, i}^{(n+1)}\right)$. Мы будем назьвать порядок малости $(G \cdot \varepsilon)^{n}$ основным. Величины, допускаюшие оценку $\left(G \cdot \varepsilon^{1+\gamma}\right)^{n}$ при $\gamma>0$ и не зависяшем от $n$, будем называть имеюшими более высокий порядок малости. В частности, как легко следует из индуктивной процедуры, другая погрешность $h_{\alpha, i}^{(n)}$ имеет более высокий порядок малости, поскольку она образуется из квадратичных (и более высоких степеней) погрешностей в формулах теории возмушений.

Далее, формула (25) показывает, что $H_{\alpha} \widetilde{\Psi}_{\alpha, i}^{(n)}-\lambda_{\alpha, i}^{(n+1)} \widetilde{\Psi}_{\alpha, i}^{(n)}$ имеет основной порядок малости, поскольку она имеет, как минимум, малость $b_{\alpha, i}^{(n)}$, но правая часть (25) на самом деле меньше из-за близости базиса $\left\{\Psi_{\alpha+m \omega, i}^{(n)}\right\}$ к ортогональному.

Теперь мы можем применить прием, описанный в начале $\S 3$. Допустим, что $m$ лежит справа от $Z\left(\Psi_{\alpha, i}^{(n)}\right)$. Положим $\varphi_{\alpha, i}^{(n)}(x)$ равной $\widetilde{\Psi}_{\alpha, i}^{(n)}(x)$ при $x \leqslant m$ и 0 при $x>m$. Тогда

$$
H_{\alpha} \varphi_{\alpha, i}^{(n+1)}(x)-\lambda_{\alpha, i}^{(n+1)}(x) \varphi_{\alpha, i}^{(n+1)}(x)=e_{\alpha, i}^{(n+1)}(x)
$$

при $x \neq m, m+1$ и $e_{\alpha, i}^{(n+1)}(x)$ не превосходит по модулю $\left(C_{6} \varepsilon\right)^{n}$. С другой стороны,

$$
\begin{aligned}
H_{\alpha} \varphi_{\alpha, i}^{(n+1)}(m)-\lambda_{\alpha, i}^{(n+1)} \varphi_{\alpha, i}^{(n+1)}(m) & =\varepsilon \Psi_{\alpha, i}^{(n+1)}(m)+e_{\alpha, i}^{(n+1)}(m+1), \\
H_{\alpha} \varphi_{\alpha, i}^{(n+1)}(m+1)-\lambda_{\alpha, i}^{(n+1)} \varphi_{\alpha, i}^{(n+1)}(m+1) & =\varepsilon \Psi_{\alpha, i}^{(n+1)}(m)+e_{\alpha, i}^{(n+1)}(m+1),
\end{aligned}
$$

где $e_{\alpha, i}^{(n+1)}(m+1), e_{\alpha, i}^{(n+1)}(m)$ удовлетворяют тем же оценкам, что и $e_{\alpha, i}^{(n+1)}(x)$. Кроме того, $\lambda_{\alpha, i}^{(n+1)}-\lambda_{\alpha, i}^{(n)}$ имеет более высокий порядок малости, поскольку $h_{\alpha, i}^{(n)}$ имеет более высокий порядок малости и $\Psi_{\alpha, i}^{(n)}$ на носителе $b_{\alpha, i}^{(n)}$ имеет основной порядок малости. 
Отсюда следует, что модуль разности двух собственных значений, полные носители которых пересекаются, удовлетворяет неравенству

$$
\left|\lambda_{\alpha, i}^{(n+1)}-\lambda_{\alpha, j}^{(n+1)}\right| \geqslant\left(\frac{C_{4} \varepsilon}{2}\right)^{n} .
$$

Положим

$$
\begin{gathered}
\widetilde{b}_{\alpha, i}^{(n+1)}(m)=-\varepsilon \Psi_{\alpha, i}^{(n+1)}(m+1), \quad \widetilde{b}_{\alpha, i}^{(n+1)}(m+1)=\varepsilon \Psi_{\alpha, i}^{(n+1)}(m), \\
\widetilde{b}_{\alpha, i}^{(n+1)}(x)=0 \text { при остальных } x
\end{gathered}
$$

и введем вектор $\widetilde{h}_{\alpha, i}^{(n+1)}$ с помощью соотношения

$$
\left(H_{\alpha}-\lambda_{\alpha, i}^{(n+1)}\right) \varphi_{\alpha, i}^{(n+1)}=\widetilde{b}_{\alpha, i}^{(n+1)}+\widetilde{h}_{\alpha, i}^{(n+1)}
$$

Наши предыдушие рассуждения показывают, что $\widetilde{h}_{\alpha, i}^{(n+1)}$ имеет более высокий порядок малости. Пусть

$$
\widetilde{\varphi}_{\alpha, i}^{(n+1)}=\varphi_{\alpha, i}^{(n+1)}+\sum_{j \neq i} \frac{\left(\widetilde{b}_{\alpha, i}^{(n+1)}+\widetilde{h}_{\alpha, i}^{(n+1)}, \Psi_{\alpha, i}^{(n+1)}\right)}{\lambda_{\alpha, i}^{(n+1)}-\lambda_{\alpha, j}^{(n+1)}}
$$

Тогда $H_{\alpha} \widetilde{\varphi}_{\alpha, i}^{(n+1)}-\widetilde{\lambda}_{\alpha, i}^{(n+1)} \widetilde{\varphi}_{\alpha, i}^{(n+1)}$ также имеет более высокий порядок малости. Поэтому $\widetilde{\varphi}_{\alpha, i}^{(n+1)}-\widetilde{\Psi}_{\alpha, i}^{(n+1)}$ также имеет более высокий порядок малости в том смысле, что каждая компонента разности имеет более высокий порядок малости. Напишем

$$
\Psi_{\alpha, i}^{(n+1)}(m+1)=\widetilde{\Psi}_{\alpha, i}^{(n+1)}(m+1)=\widetilde{\varphi}_{\alpha, i}^{(n+1)}(m)+g_{\alpha, i}^{(n+1)}(m),
$$

где $g_{\alpha, i}^{(n+1)}(m)$ имеет более высокий порядок малости. Далее, с помошью (27) при $x=m+1$

$$
\begin{aligned}
& \Psi_{\alpha, i}^{(n+1)}(m+1)=\varepsilon \Psi_{\alpha, i}^{(n+1)}(m) \sum_{j \neq i} \frac{\Psi_{\alpha, i}^{(n+1)}(m+1)}{\lambda_{\alpha, i}^{(n+1)}-\lambda_{\alpha, j}^{(n+1)}} \\
&-\varepsilon \Psi_{\alpha, i}^{(n+1)}(m+1) \sum_{j \neq i} \frac{\Psi_{\alpha, i}^{(n+1)}(m+1)}{\lambda_{\alpha, i}^{(n+1)}-\lambda_{\alpha, j}^{(n+1)}}+\widetilde{g}_{\alpha, i}^{(n+1)}(m+1),
\end{aligned}
$$

где $\widetilde{g}_{\alpha, i}^{(n+1)}(m+1)$ имеет более высокий порядок малости. Поэтому

$$
\begin{array}{r}
\Psi_{\alpha, i}^{(n+1)}(m+1)=\varepsilon \Psi_{\alpha, i}^{(n+1)}(m) \cdot\left(\sum_{j \neq i} \frac{\Psi_{\alpha, j}^{(n+1)}(m+1)}{\lambda_{\alpha, i}^{(n+1)}-\lambda_{\alpha, j}^{(n+1)}}\right)\left(1+\varepsilon \sum_{j \neq i} \frac{\Psi_{\alpha, i}^{(n+1)}(m)}{\lambda_{\alpha, i}^{(n+1)}-\lambda_{\alpha, j}^{(n+1)}}\right)^{-1} \\
+\widetilde{g}_{\alpha, i}^{(n+1)}(m+1)\left(1+\varepsilon \sum_{j \neq i} \frac{\Psi_{\alpha, j}^{(n+1)}(m+1)}{\lambda_{\alpha, i}^{(n+1)}-\lambda_{\alpha, j}^{(n+1)}}\right)^{-1} .
\end{array}
$$


Последняя формула показывает, что в главном порядке отношение

$$
\Psi_{\alpha, i}^{(n+1)}(m+1) / \Psi_{\alpha, i}^{(n+1)}(m)
$$

равно

$$
\varepsilon\left(\sum_{j \neq i} \frac{\Psi_{\alpha, j}^{(n+1)}(m+1)}{\lambda_{\alpha, i}^{(n+1)}-\lambda_{\alpha, j}^{(n+1)}}\right)\left(1+\varepsilon \sum_{j \neq i} \frac{\Psi_{\alpha, i}^{(n+1)}(m)}{\lambda_{\alpha, i}^{(n+1)}-\lambda_{\alpha, j}^{(n+1)}}\right)^{-1}
$$

и мало в силу присутствия $\varepsilon$, поскольку возникающая здесь дробь принимает в типичной ситуации значения порядка 1.

Мы можем написать

$$
\begin{array}{r}
\frac{\Psi_{\alpha, i}^{(n+1)}(m+k)}{\Psi_{\alpha, i}^{(n+1)}(m)}=\prod_{s=1}^{k}\left[\left(\varepsilon \sum_{j \neq i} \frac{\Psi_{\alpha, j}^{(n+1)}(m+s+1)}{\lambda_{\alpha, i}^{(n+1)}-\lambda_{\alpha, j}^{(n+1)}}\right)\left(1+\varepsilon \sum_{j \neq i} \frac{\Psi_{\alpha, j}^{(n+1)}(m+s)}{\lambda_{\alpha, i}^{(n+1)}-\lambda_{\alpha, j}^{(n+1)}}\right)^{-1}\right. \\
\left.+\widetilde{g}_{\alpha, i}^{(n+1)}(m+s+1)\left(1+\varepsilon \sum_{j \neq i} \frac{\Psi_{\alpha, j}^{(n+1)}(m+s)}{\lambda_{\alpha, i}^{(n+1)}-\lambda_{\alpha, j}^{(n+1)}}\right)^{-1}\right] .
\end{array}
$$

Из этой формулы вытекает экспоненциальное убывание приближенных с.ф. вдали от с.н. Однако при использовании (29) следует иметь в виду, что возможны ситуации, когда знаменатель $1+\varepsilon \sum_{j \neq i} \frac{\Psi_{\alpha, j}^{(n+1)}(m+s)}{\lambda_{\alpha, i}^{(n+1)}-\lambda_{\alpha, j}^{(n+1)}}$ аномально мал. Тогда и отношение $\frac{\Psi_{\alpha, j}^{(n+1)}(m+s+1)}{\Psi_{\alpha, j}^{(n+1)}(m+s)}$ аномально велико. Поскольку $H_{\alpha} \Psi_{\alpha, i}^{(n+1)}-\lambda_{\alpha, i}^{(n+1)} \Psi_{\alpha, i}^{(n+1)}$ имеет более высокий порядок малости, то отношение $\frac{\Psi_{\alpha, j}^{(n+1)}(m+s+1)}{\Psi_{\alpha, j}^{(n+1)}(m+s)}$ аномально близко $\mathrm{K} 1$.

Это означает, что знаменатель $1+\varepsilon \sum_{j \neq i} \frac{\Psi_{\alpha, j}^{(n+1)}(m+s-1)}{\lambda_{\alpha, i}^{(n+1)}-\lambda_{\alpha, j}^{(n+1)}}$ аномально велик, а произведение двух рядом стоящих знаменателей имеет порядок 1.

Далее, основной вклад в дробь

$$
\frac{\sum_{j \neq i} \frac{\Psi_{\alpha, j}^{(n+1)}(m+s)}{\lambda_{\alpha, i}^{(n+1)}-\lambda_{\alpha, j}^{(n+1)}}}{1+\varepsilon \sum_{j \neq i} \frac{\Psi_{\alpha, j}^{(n+1)}(m+s)}{\lambda_{\alpha, i}^{(n+1)}-\lambda_{\alpha, j}^{(n+1)}}}
$$

вносят приближенњые с.ф., которые сосредоточены около точки $m+s$. Поэтому эту дробь естественно записать как $\Theta^{(n+1)}\left(\lambda_{\alpha, i}^{(n+1)} ; \alpha+(m+s) \omega\right)$ и к произведению (29) применить эргодическую теорему. Из всех этих рассуждений вытекает свойство $7^{\circ}$ c.ф̆. (cм. $\S 1)$. 
Рассмотрим теперь более подробно расшепление приближенных с.з. в резонансной ситуации. Если в (27) сомножители имеют основной порядок малости, то и расшепление приближенных с.з. имеет основной порядок малости. Его величина определяет размер запрещенной зоны, которая возникает на $n$-м шаге. В принципе возможно, что один из сомножителей $\Psi_{\alpha, i}^{(n+1)}(m-1)$ или $\Psi_{\alpha, j}^{(n+1)}\left(m_{0}\right)$ аномально мал. Тогда возникшие приближенные с.ф. принимают в точке $m_{0}$ аномально малые значения, что возможно при нетипичных $\alpha$. В таком случае следует перейти от $m_{0} \mathrm{~K} m_{0}-1$, продолжить $\Psi_{\alpha, j}^{(n)}$ еше на один шаг до $m_{0}-1$, пользуясь формулами нерезонансного случая, а вместо $\Psi_{\alpha, j}^{(n)}$ рассмотреть ее ограничение на $F Z\left(\Psi_{\alpha, i}^{(n)}\right) \backslash m_{0}$. Формулы теории возмущений будут при этом применимы и $\Psi_{\alpha, i}^{(n+1)}\left(m_{0}-1\right), \Psi_{\alpha, j}^{(n)}\left(m_{0}\right)$ будет иметь основной порядок малости. После этого можно использовать предыдущие рассуждения. Отсюда вытекают также оценки $\left.\left.\mathrm{i}_{0}\right)-\mathrm{i}_{4}\right)$ и $\left.\left.\mathrm{j}_{1}\right)-\mathrm{j}_{3}\right)$ с подходящими константами $b_{1}, B_{2}, B_{3}$.

Обсудим теперь вопрос о стремлении базиса $\bigcup S^{m} \Psi_{\alpha+m \omega, i}^{(n)}$ к ортогональному и о малости скалярных произведений $\left(\Psi_{\alpha, i}^{(n)}, \Psi_{\alpha, j}^{(n)}\right)=\xi_{\alpha, i, j}^{(n)}, i \neq j$. Мы имеем

$$
\left(H_{\alpha} \Psi_{\alpha, i}^{(n)}, \Psi_{\alpha, j}^{(n)}\right)=\lambda_{\alpha, i}^{(n)}\left(\Psi_{\alpha, i}^{(n)}, \Psi_{\alpha, j}^{(n)}\right)+\left(b_{\alpha, i}^{(n)}+h_{\alpha, i}^{(n)}, \Psi_{\alpha, j}^{(n)}\right)
$$

В силу самосопряженности $H_{\alpha}$

$$
\left(H_{\alpha} \Psi_{\alpha, i}^{(n)}, \Psi_{\alpha, j}^{(n)}\right)=\left(\Psi_{\alpha, i}^{(n)}, H_{\alpha} \Psi_{\alpha, j}^{(n)}\right)=\lambda_{\alpha, j}^{(n)}\left(\Psi_{\alpha, i}^{(n)}, \Psi_{\alpha, j}^{(n)}\right)+\left(b_{\alpha, i}^{(n)}+h_{\alpha, i}^{(n)}, \Psi_{\alpha, i}^{(n)}\right) .
$$

Правая часть последнего соотношения имеет основной порядок малости, поскольку $h_{\alpha, j}^{(n)}$ имеет основной порядок малости, и малость $\left(\Psi_{\alpha, i}^{(n)}, b_{\alpha, j}^{(n)}\right)$ уменьшается еще сильнее при удалении с.н. $\Psi_{\alpha, i}^{(n)}$ от носителя $b_{\alpha, j}^{(n)}$. Если $\Psi_{\alpha, i}^{(n)}, \Psi_{\alpha, j}^{(n)}$ не получились в результате применения резонансных формул к паре приближенных с.ф., то $\left|\lambda_{\alpha, i}^{(n)}-\lambda_{\alpha, j}^{(n)}\right| \geqslant$ $\left(\frac{1}{2} C_{4} \varepsilon\right)^{n}$. Значение константы $C_{4}$ определяет размер резонансных зон. Мы выберем ее достаточно большой так, чтобы из последнего соотношения вытекало

$$
\left|\xi_{\alpha, j}^{(n)}\right| \leqslant\left(\frac{C}{C_{4}}\right)^{4}
$$

где $C$ - константа, участвующая в экспоненциальной оценке убьвания приближенных c.фp.:

$$
\left|\Psi_{\alpha, i}^{(n)}(x)\right| \leqslant(C \varepsilon)^{\operatorname{dist}\left(x, Z\left(\Psi_{\alpha, j}^{(n)}\right)\right)} .
$$

Если приближенные с.ф. $\Psi_{\alpha, i}^{(n)}, \Psi_{\alpha, j}^{(n)}$ получились в результате применения резонансных формул теории возмушений, то они ортогональны по построению.

Значения остальных постоянных, введенных при описании свойств ЛА, легко вытекают из конструкции.

Эта работа быланачата во время посещения осенью 1997 года отделения математики Стенфордского Университета и плодотворных бесед с И. Катцнельсоном и Д. Орнстейном. Я благодарен им, а также Е. И. Динабургу и В. Шлагу за полезные обсуждения и NSF (грант DMS-9706794) и РФФИ (грант 96-01-0037) за финансовую поддержкy. 


\section{СПИСОК ЛИТЕРАТУРЫ}

[1] Синай Я. Г. Предельное поведение одномерного случайного блуждания в случайной среде // Теория вероятн. и ее примен. 1982. Т. 27. № 2. С. 247-258.

[2] Fröhlich J., Spencer T., Wittwer P. Localization for a class of one-dimensional quasi-periodic Schrödinger operators // Comm. Math. Phys. 1990. V. 132. № 1. P. 5-25.

[3] Eliasson L.H. Discrete one-dimensional quasi-periodic Schrödinger operators with pure point spectrum // Acta Math. 1997. V. 179. № 2. P. 153-196.

[4] Sinai Ya. G. Anderson localization for the one-dimensional difference Schrödinger operator with quasi-periodic potential // J. Statist. Phys. 1987. V. 46. P. 861-909.

[5] Goldstein M. Anderson Localization for Quasi-Periodic Schrödinger Equation // Preprint, 1998.

[6] Eliasson L. H. Floquet solutions for the 1-dimensional quasi-periodic Schrödinger equation // Comm. Math. Phys. 1992. V. 146. № 3. P. 447-482.

[7] Moser Ju., Pöschel J. An extension of a result by Dinaburg and Sinai on quasi-periodic potentials // Comment. Math. Helvet. 1984. V. 59. P. 39-85.

Отделение математики Принстонского университета

Поступила в редакцию

и Институт теоретической физики им. Л. Д. Ландау РАН

22.09.1998 